\title{
Modified symbiotic organisms search for structural optimization
}

\author{
Sumit Kumar ${ }^{1} \cdot$ Ghanshyam G. Tejani $^{2}$ [D $\cdot$ Seyedali Mirjalili ${ }^{3}$
}

Received: 1 October 2018 / Accepted: 12 November 2018

(c) Springer-Verlag London Ltd., part of Springer Nature 2018

\begin{abstract}
The structural dynamic response predominantly depends upon natural frequencies which fabricate these as a controlling parameter for dynamic response of the truss. However, truss optimization problems subjected to multiple fundamental frequency constraints with shape and size variables are more arduous due to its characteristics like non-convexity, nonlinearity, and implicit with respect to design variables. In addition, mass minimization with frequency constraints are conflicting in nature which intricate optimization problem. Using meta-heuristic for such kind of problem requires harmony between exploration and exploitation to regulate the performance of the algorithm. This paper proposes a modification of a nature inspired Symbiotic Organisms Search (SOS) algorithm called a Modified SOS (MSOS) algorithm to enhance its efficacy of accuracy in search (exploitation) together with exploration by introducing an adaptive benefit factor and modified parasitism vector. These modifications improved search efficiency of the algorithm with a good balance between exploration and exploitation, which has been partially investigated so far. The feasibility and effectiveness of proposed algorithm is studied with six truss design problems. The results of benchmark planar/space trusses are compared with other meta-heuristics. Complementarily the feasibility and effectiveness of the proposed algorithms are investigated by three unimodal functions, thirteen multimodal functions, and six hybrid functions of the CEC2014 test suit. The experimental results show that MSOS is more reliable and efficient as compared to the basis SOS algorithm and other state-of-the-art algorithms. Moreover, the MSOS algorithm provides competitive results compared to the existing meta-heuristics in the literature.
\end{abstract}

Keywords Natural frequency $\cdot$ Truss optimization $\cdot$ Meta-heuristics $\cdot$ Adaptive mechanism $\cdot$ Exploration $\cdot$ Exploitation

\section{Introduction}

The design of optimum structure is an active research area due to its wide range of applications in bridges, towers, pylons, roof supports, building exoskeletons, space

Ghanshyam G. Tejani

p.shyam23@gmail.com

Sumit Kumar

sumit21sep1990@gmail.com

Seyedali Mirjalili

ali.mirjalili@gmail.com

1 Department of Mechanical Engineering, GPERI, Gujarat Technological University, Ahmedabad, Gujarat, India

2 Department of Mechanical Engineering, School of Technology, GSFC University, Vadodara, Gujarat, India

3 School of Information and Communication Technology, Griffith University, Nathan Campus, Brisbane, QLD 4111, Australia structures, and industries. Basically, truss is a framework of flexible members pin connected at joint called node intended to withstand purely axial loads over a large span of space. Formerly, structural optimization was a concept of optimal design of load-carrying mechanical structures only. In emerging world, structural optimization is a broad concept due to diversity of aim and possible constraints, causes tremendous variation of results. Basically, objective of structural optimization is to make structures slender with minimum weight. Consequently, optimal design became a progressive area of research in search and optimization field today.

In most of dynamic truss optimization problems, the response highly depends upon the natural frequency and mode shape of structure. Therefore, to avoid condition like resonance, frequency constraints become inevitable in formulation of truss optimization problems subjected to dynamic loads. Mass optimization is considered to be challenging with inclusion of frequency constraints. Frequency 
constraints usually conflicts with lower bounded mass optimization, [16]. In addition, regarding design variables, frequency constraints are more implicit, non-linear, and non-convex, [81]. Therefore, gradient-based methods are inappropriate for these types of optimization problems as they have complex programming approach and required additional time for computation. Furthermore, for optimal solution they required good starting point, Kaveh and Talatahari [25]. Due to premature convergence and above demerits, drift is towards nature-based meta-heuristics (MHs) for scholars nowadays as they have leverage with respect to classical MHs. Noilublao and Bureerat [38] proposed an integrated design technique for simultaneously sizing, topology, and shape optimization of truss structure with frequency constraints. Results showed population-based incremental learning MHs are better for natural frequency constrained optimization problems. Gomes [16] attempted to overcome non-linearity associated with shape and size optimization of truss by accounting the particle swarm optimization (PSO) algorithm as an optimization engine with frequency constraints.

Kaveh and Zolghadr [26] suggested an algorithm termed as Charged System Search with its modified version for optimization of structural truss including frequency constraints. Miguel and Miguel [36] suggested the use of two MHs, Firefly Algorithm and Harmony Search, for solving shape and size optimization of trusses simultaneously subjected to multiple natural frequency constraints. Furthermore, [27] introduced a concept of hybridization between the Charged System Search and Big Bang-Big Crunch algorithms with natural frequency constraints, which are proficient to identify local optima trap. In addition, [28] investigated the topology optimization of structural truss with inclusion of buckling, displacement, stress, and frequency constraints. Moreover, [29] comes up with a new algorithm called as Democratic PSO for structural optimization with inclusion of frequency constraints to alleviate the premature convergence phenomenon of the basic PSO algorithm. Farshchin et al. [12] proposed a multi-class Teaching-Learning-Based approach (MC-TLBO) with frequency constraints for structure optimization to increases the exploration capability and enhancing the search efficiency.

Kaveh and Zolghadr [30] developed a multi-agent meta-heuristic $(\mathrm{MH})$ called Tug of War Optimization to optimize size and shape of structural trusses subjected to frequency constraints. Kaveh and Ghazaan [20] employed physically inspired non-gradient algorithm called Vibrating Particles System (VPS) for structural optimization subjected to frequency constraints. Kaveh and Mahdavi [23] utilized colliding bodies' optimization (CBO) for truss structure optimal design subjected to dynamic constraints. Furthermore, Kaveh and Mahdavi [24] proposed two-dimensional version of $\mathrm{CBO}$, with advancement in its algorithm formulation which remain untouched in one-dimension CBO. The results show better search performance within minimum computational time. Savsani et al. [42] comes up with an improved form of the Teaching-Learning-Based Optimization algorithm for optimization of trusses topology considering different static and dynamic constraints. Wang et al. [53] presented an algorithm for 3-D truss structure optimization with multiple constraints on its natural frequencies. Wei et al. [54] proposed a niche hybrid parallel genetic algorithm (NHPGA) for simultaneous size and shape optimization of structural trusses subjected to numerous frequency constraints. Here, NHPGA combined the advantage of simplex search, parallel computing and genetic algorithm with niche technique for getting effective and efficient solution. Zuo et al. [59] for obtaining global solution and to speed up convergence of truss optimization problems used genetic algorithm and hybrid optimality criterion algorithm with multiple frequency constraints.

Kaveh et al. [21] applied Dolphin Echolocation algorithm for optimization of truss structure with frequency constraints. Efficiency of algorithm was compared with hybrid MHs. Kaveh and Ghazaan [83] applied hybridized algorithm for structural optimization with multiple natural frequency constraints. They applied Aging Leader and Challengers with PSO and harmony-based search mechanism with PSO for optimization search problem with natural frequency constraints. Due to its auto tuned capability it has high convergence ability with better stability of structure. Mortazavi and Togan [37] demonstrate the competitive edges of integrated PSO in optimization of truss structures with integration of the weighted particle concept and fly-back mechanism under multiple frequency constraints with sizing and layout variables. Savsani et al. [43] considered four modified MHs to solve distinct obstacles of optimization.

Recently many MHs showed the superiority with respect to gradient-based algorithms as they do not require gradient-based calculation [33]. MHs include the natural evolutionary process like the evolutionary algorithm proposed by Fogel [13], De Jong [5], Koza [32] and the Genetic Algorithm (GA) proposed by Goldberg and Holland [15], and animal behavior, e.g., Tabu search proposed by Glover [14], Ant algorithm proposed by Dorigo et al. [7], PSO proposed by Eberhart and Kennedy [8], the Artificial Bee Colony algorithm proposed by Karaboga and Basturk [17], Firefly Algorithm by Yang [56], Cuckoo Search by Yang and Deb [57], Dolphin Echolocation by Kaveh and Farhoudi [18] and the physical annealing process like simulated annealing proposed by Kirkpatrick et al. [31] and more like Harmony Search by Geem et al. [78], Big Bang-Big Crunch by Erol and Eksin [9], Water Cycle Algorithm by Eskandar et al. [10], and Ray Optimization 
by Kaveh and Khayatazad [22]. However, above MHs need extra controlling parameters and also not easy to use. For example, GA needs mutation and crossover rates, while PSO requires inertia weight, social, and cognitive parameters. Furthermore, many MHs need to perform parameter tuning, where improper tuning related to algorithm-specific parameters might increase computational time and produce local optima solutions. They show inadequacy in robustness and generalization.

The resemblance in MHs is that they incorporate rules and randomness to imitate organic phenomena. MHs generally employs various iterative process to find efficient optimal solution in search space with integration of exploration and exploitation notion to escort a minor heuristic with learning strategies [39]. However, Wolpert and Macready [55] rationalized that it is impossible to solve all optimization problem with one MH. Therefore, a recent efficient and high performing $\mathrm{MH}$ is required to solve complex optimization problems.

Symbiotic Organisms Search (SOS) is a recent algorithm which mimics the strategies of mutualism, commensalism, and parasitism encouraged by natural synergy between organisms in ecosystems. SOS has better exploitation capability across benchmark problems due to ease in adjustability of its common parameters which make it simple to operate as well as its ability of merit solution generation within fewer iterations [3]. Different studies acknowledged the novelty of SOS than other competing MHs due to its simplicity of implementation with ability of better exploration and exploitation using minimum controlling parameters. Many scholars also compared its performance for various benchmark functions and optimization problems across other MHs with outcome of better performances [60-64]. Moreover, lots of research demonstrated the superiority of SOS over other MHs like Panda and Pani [65] united the SOS with Augmented Lagrange Multiplier method to solve constrained optimization problems. This fusion enhances the result accuracy within lower run time compared to other MHs. Prayogo et al. [66] applied SOS with an improvement in the parasitism phase for optimization of resource leveling of construction project. The experimental result shows the better quality solution in comparison with existing optimization models. Similar ascendancy conduct of SOS has been detected by Yu et al. [58] while application in capacitated vehicle routing problem. Authors proposed six improved version of SOS with inclusion of two new interaction strategies, namely competition and amensalism in the basic SOS algorithm for performance enhancement. Likewise, a new hybrid artificial intelligence system, SOS-LSSVR (least squares support vector regression), has been proposed to predict the permanent deformation potential of asphalt pavement mixtures (Cheng et al. [4]). Proposed study is able to achieve better accuracy than all other comparative measures which manifest the outperformance of SOS-based system over other methods. Abdullahi et al. [63] presents a Discrete Symbiotic Organism Search (DSOS) for optimal scheduling of tasks on cloud resources. Simulation results revealed that DSOS outperforms PSO which is one of the most popular heuristic optimization techniques used for task scheduling problems. DSOS converges faster when the search gets larger which makes it suitable for large-scale scheduling problems. Banerjee and Chattopadhyay [67] proposed a modified SOS to design an improved 3 dimensional turbo code. SOS a novel powerful MH optimization technique is also proposed for the first time to solve the load frequency control problem [68]. Results show that the dynamic stability of the concerned power system effective enhancement with SOS. Furthermore, Prayogo et al. [72] in their doctoral dissertation compared the effectiveness of SOS in solving various civil engineering and benchmark problems. Simulation results demonstrate that SOS is significantly more effective and efficient than the other algorithms present in the literature. Analogously, lots of research [69-71, $73,74]$ are available in the literature which flourishes the supremacy of SOS over other MHs.

Few studies demonstrated the supremacy of adaptive strategies over fixed search mechanism for quality solution and convergence search which tune the balance between exploration and exploitation $[11,50]$. For improvisation in exploration and exploitation potential of the basic SOS algorithm, Tejani et al. [46] introduced an adaptive benefit factors which result into more-reliable and balanced solution of dynamics structures subjected to frequency limits. Tejani et al. [48] suggested a modified and improved version of SOS to overcome non-convexity, non-linearity drawback of basic algorithms with renovation in search performance. Ezugwu et al. [11] presents SOS with simulated annealing (SA) to solve the traveling salesman problems. Results demonstrate better solution convergence, minimum execution time with minimum deviation of solution from best solution. Yu et al. [58] presented improved SOS with two new interaction strategies called competition and amensalism. Liao and Kuo [35] develop five new discrete SOS algorithms. Tran et al. [51] introduced opposition multiple objective SOS for scheduling repetitive projects. Similarly, Tejani et al. [52] utilized multiobjective adaptive symbiotic organisms search for truss optimization problems. Despite of various advantages, above-mentioned algorithms lacks in key components. In addition, in these algorithms if their exploration capacity is superior then exploitation capacity decreases comparatively and vice versa. Furthermore, they untouched the parasitism phase which leads to problem of large computational time for getting solution of optimization process. Therefore, for better result a good balance 
between exploration and exploitation capacity of algorithm is required.

Non-linearity and non-convexity characteristics of shape and size variables with frequency limits, cause optimization problem more challenging. SOS incompetency for dynamic structural optimization with natural frequency limits has been displayed by many scholarly findings [79], (Crepinsek et al. 2013). Research depicts the heuristic nature of benefit factor, i.e., 1 or 2 in the basic SOS algorithm, which represents the organism interaction as partial or complete only. However, the benefit factor which organism may get could be in any scale between 1 and 2. The SOS algorithm though efficient in solving complex optimization and discrete engineering problems, still has high probability of plunging into local optimum [48]. The SOS algorithm also lags in iterative process of size of population selection. The reason is there are instances when selecting a small population size yields a better result than a large population size and vice versa. For example, if the algorithm is stopped too early, the approximation of the solution might not be close to the targeted global optimum and prolonging the simulation might as well incur unnecessary scale up in the computational effort. Ezugwu et al. [11] have shown the improvement in efficiency of algorithm via reducing the solution completion time, introduce diversity in the search process and avoid premature convergence through integration of local search strategy into basic SOS. Above researches motivate the researchers to extend their research by incorporating additional improvements into SOS using hybridization techniques, to include other MHs such as SA and test its performance on different set of larger problem instances. In addition, many researches show the requirement of improvement in basic SOS for discrete and continuous optimization problems [6]. Few research scholars show the possibility of better trade of between exploration and exploitation capability of SOS with modification in mutualism and parasitism phase. Miao et al. [84] presents a modified SOS algorithm based on the simplex method to solve the path planning problems. Results shows, modification of SOS leads to faster convergence speed, higher precision, and stronger robustness than the main algorithm. Numerous scholars show that metamorphosis lead to increase in the diversity of the population and improvement in the ability of the algorithm to explore and exploit, as well as preventing the algorithm from prematurely finding the local optimal solution. Moreover, modification testing on various benchmark problems shows enhancement in accuracy and robustness with stronger convergence speed than other algorithms.

Despite of aforementioned advantages, the SOS algorithm inadequacy for dynamic structural optimization with natural frequency limits has been uncovered by many scholarly findings. Moreover, it is practically not possible to forecast the influence of the adaptive operators for different real life applications [49]. As per the No Free Lunch theorem, it is impossible to solve all optimization problems with one algorithm. Furthermore, the SOS algorithm is a recently developed algorithm and it is always interesting to investigate different modification that can improve the performance of the algorithm. These proficiencies and prospects encouraged us to formulate an adaptive SOS algorithm with frequency constraints and to investigate its effect on dynamic structural optimization problems. These inadequacies like lack of efficient exploration, balanced search with good success rate and less computation time, give impetus to formulate a modified SOS (MSOS) algorithm and to examine its effect on different structural optimization problems.

In other words, a good balance of exploration and exploitation is essential to avoid local solutions and find an accurate estimation of the global optimum for a given optimization problem. To alleviate these drawbacks, MSOS is equipped with adaptive benefit factor to tune the balance between exploration and exploitation during mutualism phase and an improved parasitism phase to boost exploitation capability during parasitism phase of SOS.

The rest of the paper is organized as follows: Sect. 2 presents the SOS algorithm. The improved SOS is proposed in Sect. 3. The problem is formulated in Sect. 4 and solved using the modified SOS in Sect. 5. Moreover, Sect. 6 includes verification of MSOS performance with various functions of CEC 2014 test suits. Finally, Sect. 7 concludes the work and suggest future directions.

\section{The symbiotic organisms search algorithm}

In nature, an essential relationship is maintained by many organisms for survival and growth. These relations may be beneficial or harmful sometimes. In symbiotic relationship species live together for mutual benefit and survival, which make it different from other regular interaction. Considering the natural interaction between the organism for survival in ecosystem Cheng and Prayogo [3] introduced a compelling and modest meta-heuristic algorithm called SOS which simulates the interactive behavior among organism seen in nature. Symbiosis describes close interaction between two or more species like snail and tortoise, human and dog, Lichen (algae and fungus), etc. In either of case, each organism helps together in existence and continuation while prevailing relationship.

Unlike other MHs which need extra optimization parameters SOS algorithm required only general controlling parameters like total no of function evaluations (FE) for its operation and population size (number of organism). For example, GA needs mutation, crossover, and rates, while 
PSO requires inertia weight, social, and cognitive parameters [46]. On the other hand, SOS does not need algorithmspecific governing factors and is simply organized and easy to use. It is also robust and generalized. This is considered an advantage over competing algorithms, because SOS does not need to perform parameter tuning. Improper tuning related to algorithm-specific parameters might increase computational time and produce local optima solutions.

Symbiotic relationship can be divided into three phases: mutualism, commensalism and parasitism.

Mutualism: In this phase, the organism tries to find the best solution in mutual benefit interaction with other to find out the optimum value greater than previous one- a classic example is the interaction between bees and flowers;

Commensalism: In this phase, organisms with best functional value is trying to find without affecting the other possible solution (organism) nearby- an example is the relationship between remora fish and sharks; and

Parasitism: In this phase, best solution is obtained with suffering of other organism- an example is the plasmodium parasite, which uses its relationship with the anopheles mosquito to transfer between human hosts.

The three phases are adopted from the most common symbioses used by organisms to increase their fitness and survival advantage over the long term. In this optimization algorithm, the better solution can be achieved by the symbiotic relations between the current solution vector and either the other randomly selected solution or the best solution from the current population. The phases are repeated until the stopping criterion is achieved.

The detailed discussion of all three phases and modification of SOS is explained in the subsequent sections:

\subsection{The mutualism phase}

When both organisms of different species get individual benefit from interaction then it is called a mutualism. A common example of mutualism is the relationship between bees and flowers, where both organisms acquire benefits from each other. Bees obtained sweet food source secreted by the flowers (called nectar) through visiting different flowers which benefit them to produce honey. On the other hand, while travelling bees transfer pollen grains from one plant to another which facilitate pollination in flowers (benefit to flowers).

In mutualism phase, a sequentially selected organism $\left(X_{i}\right)$ in the ecosystem interacts with another random member say $\left(X_{j}\right)$, where $i \neq j$. Both organisms engage in a mutuality relationship with the goal of increasing mutual survival advantage in the ecosystem. They are interdependent and each gain benefit from other. New candidates are calculated based on interact with the motive of mutual survival advantage in the ecosystem as per Eqs. $(1,2)$ :
$X_{\text {inew }}=X_{i}+\operatorname{rand}(0,1) \times\left(X_{\text {best }}-\right.$ Mutual_Vector $\left.\times B F_{1}\right)$,

$X_{\text {jnew }}=X_{j}+\operatorname{rand}(0,1) \times\left(X_{\text {best }}-\right.$ Mutual_Vector $\left.\times \mathrm{BF}_{2}\right)$

where

Mutual_Vector $=\frac{X_{i}+X_{j}}{2}$

$\mathrm{BF}_{1}=1+\operatorname{round}[\operatorname{rand}(0,1)]$

$\mathrm{BF}_{2}=1+\operatorname{round}[\operatorname{rand}(0,1)]$

The following observations on the mutualism mathematical model can be made:

- $\operatorname{rand}(0,1)$ in Eqs. $(1,2)$ is a vector resenting random numbers between 0 and 1 ;

- Mutual_Vector in Eq. (3) represents the relationship attributes between organisms $X_{i}$ and $X_{j}$;

- $X_{\text {best }}$ represents the organism with the current highest state of adaptation to the ecosystem;

- Organism $X_{i}$ might benefit significantly when interacting with organism $X_{j}$; at the same time, organism $X_{j}$ might benefit only slightly when interacting with organism $X_{i}$.

\subsection{The commensalism phase}

Living organisms in nature generally react and live with one another for adaptation in various forms. Commensalism is an interesting relationship one out of them. In this type of symbiotic relationship, one will get benefit, while the other will remain unaffected or not harmed. Some time it is also called as one-sided symbiotic relationship. The welfare can be in any form like seed dispersal, transportation, food, and shelter. One of the examples is remora and shark in which remora remains attached with shark for getting leftover food but shark remain neutral.

In this phase, the best from mutualism phase taken as an input and an organism is arbitrarily selected from ecosystem which tries to communicate with others. Unlike mutualism phase, modification is only for one organism and other remain as previous one, whereas overhaul is only when new fitness value is superior to previous. The new organism is presented in Eq. (6):

$X_{\text {inew }}=X_{i}+\operatorname{rand}(-1,1) \times\left(X_{\text {best }}-X_{j}\right)$

Some observations on the commensalism mathematical model can be made:

- $\operatorname{rand}(-1,1)$ in Eq. (6) is a vector of random numbers between -1 and 1 ; 
- $X_{\text {best }}$ reflects the current highest state of adaptation to the ecosystem, similar to that used in the mutualism phase;

- Organism $X_{i}$ is updated to $X_{\text {inew }}$ only if its new fitness is better than its pre-interaction fitness; and

- For each organism $X_{i}$, this interaction counts for one function evaluation.

\subsection{The parasitism phase}

In parasitism phase, parasite gets only benefit while living in host body, causing harm to host from its long-term interaction. Here one organism gets complete benefit, while other gets eliminated completely from the system. One of the examples is plasmodium parasite which use Anopheles mosquito to enter into human body which act like host. The parasite flourishes and reproduces inside of host and human body suffers disease which may result into death.

After the commensalism phase is completed, the organism $X_{i}$ again randomly selects a new organism from the ecosystem, organism $X_{j}$. In this phase a design vector $\left(X_{i}\right)$ of " $i$ " population organism play role of anopheles mosquito. Here anopheles mosquito creates an artificial vector called "Parasite Vector". During the interaction, Parasite Vector tries to kill host $X_{j}$ and replace it in the ecosystem. Organism $X_{i}$ may gain a benefit, because, by cloning Parasite Vector, its influence in the ecosystem may increase, whereas $X_{j}$ may suffer and die.

The Parasite Vector is presented in Eq. (7):

Parasite Vector $=\left\{\begin{array}{lr}X_{j} & \text { if } 0.5 \leq \operatorname{rand}(0,1) \\ L B+\operatorname{rand}(0,1) \times(U B-L B) \text { Otherwise }\end{array}\right.$

where LB and UB represents the solution lower bound and upper bound, respectively.

The creation of Parasite Vector is described as follows:

1. An initial Parasite Vector is created in the search space by duplicating organism $X_{i}$; some decision variables from the initial Parasite Vector are modified randomly to differentiate Parasite Vector from organism $X_{i}$;

2. A random number is created within a range from one to the number of decision variables, representing the total number of modified variables;

3. The location of the modified variables is determined stochastically using a uniform random number, which is generated for each dimension; if the random number is less than 0.5 , the variable is modified; otherwise, it stays the same; and

4. The variables are modified using a uniform distribution within the search space and Parasite Vector is ready for the parasitism phase.
Both Parasite Vector and organism $X_{j}$ are then evaluated to measure their fitness. If Parasite Vector has a better fitness value, it kills organism $X j$ and assumes its position in the ecosystem. If thefitness value of $X_{j}$ is better, $X_{j}$ has immunity from the parasite and Parasite Vector can no longer live in that ecosystem. For each organism $X_{i}$, this interaction counts for one function evaluation.

\subsection{Stopping criterion}

The optimization process terminates when a user-set stopping criterion is met. This criterion is often set as the maximum iteration number $\left(g_{\max }\right)$ or the maximum number of function evaluations $\left(F E_{\max }\right)$. The optimal solution can be identified after search process termination.

\section{Modification in the SOS algorithm}

Success rate of most of the population-based optimization algorithms depends on the equilibrium between exploration and exploitation abilities significantly. Exploration represents the ability of algorithm for global search that strongly affects the accuracy of obtained optimal solution. Whereas exploitation signifies the local search potential, this plays an important role in impacting on the convergence of the optimization algorithm. Obviously, if the exploration capacity is superior to the other, a global optimal solution can be achieved, yet the convergence is slow. This is due to the fact that the algorithm must require a remarkable amount of computational cost for seeking an optimal solution in a given whole domain. Conversely, the algorithm converges quickly, but optimal solutions may occur. Therefore, provided that the above two abilities are adjusted to gain a better balance, the solution accuracy and the convergence speed can be obtained simultaneously. Although the original SOS algorithm is good at the global search capability, the limitation on the computational cost is survived. As observed, both mutualism and commensalism phases improve the exploitation ability of the algorithm, while the parasitism phase contributes to the exploration capability. Nevertheless, the last phase requires a considerable amount of computational cost to sever its search process. If the parasitism phase is eliminated to save computational cost, the SOS approach is easily trapped at local solutions. Therefore, the other phases must be refined to preserve the balance between exploitation and exploration capabilities.

To overcome the aforementioned imperfections of the original SOS approach, a MSOS algorithm is proposed in this study. 


\subsection{Modification in mutualism phase}

Various research applications proved that the structural optimization with adaptive control algorithm has dominance in solving single and multiobjective problems with inclusion of desired properties like good convergence, superior search ability and harmony between exploration and exploitation. Adaptive control mechanisms usually advance the effectiveness of the algorithm and tune the balance between exploration and exploitation abilities [49]. In this respect, many studies have been proposed by employing various adaptive control approaches in various MHs. Piotrowski [41] used the concept of global and local mutation operators to propose an adaptive memetic differential evolution (DE) algorithm, whereas a strategically adaptive version of a DE was proposed by Bureerat and Pholdee [85]. Li and Yin [34] proposed a modified cuckoo search algorithm using an adaptive parameter setting to enhance the diversity of the population. Shan et al. [44] used adaptive control on an artificial bee colony algorithm to improve the performance. Yi et al. (2016) employed an adaptive DE based on fitness function value. Tejani et al. [47] modified a teaching-learning-based optimization using an adaptive teaching factor. Many studies to develop algorithms with adaptation for multiobjective optimization are also found in the literature. Similarly, Tejani et al. [52] utilized multiobjective adaptive symbiotic organisms search for truss optimization problems. Bingul [2] proposed an adaptive GA with dynamic fitness function for multiobjective design problems. Ou-Yang et al. [86] used a self-adaptive-velocity PSO. Pham [87] presented adaptive directional mutation to enhance DE.

On the other hand, SOS is a newly discovered algorithm which encourage investigator to enhance the algorithm performance with further modification in it. Moreover, research shows that it is impossible to find an algorithm which is able to solve all optimization problems individually, so always modification is needed in optimization algorithm [49]. In addition, SOS is new player in this arena, so it always has a good possibility of improvement in its performance.

Research depicts the heuristic nature of benefit factor, i.e., 1 or 2 in Mutualism phase of basic SOS algorithm, which represents the organism interaction as partial or complete only. However, benefit factor is the key component in influencing the Mutual_Vector in mutualism phase of SOS. The benefit factors $\left(\mathrm{BF}_{1}\right.$ andBF $\left.{ }_{2}\right)$ are determined stochastically as either 1 or 2 (Eqs. 4 and 5) in basic SOS algorithm, indicating whether an organism partially or fully benefits from the interaction. These Benefit Factors is heuristic in nature, because in nature one organism can receive partial or full benefit than other. The level of benefit interaction, i.e., full or partial is represented by these factors. Organisms evolve to a fitter version only if their new fitness is better than their pre-interaction fitness; if so, the old $X_{i}$ and $X_{j}$ are replaced by $X_{\text {inew }}$ and $X_{\text {jnew }}$; this mechanism is similar to greedy selection; and for each organism $X_{i}$, this interaction counts for two function evaluations.

In other words, the organism $X_{i}$ and $X_{j}$ can get partially and fully benefit from mutual vector. Which means when lower value of benefit factor is there then algorithm search will be fine with small step, but the convergence speed of the algorithm decreases. Similarly, if larger value of benefit factor considered the search get speed up skipping nearby value which reduces exploitation capacity of algorithm. However, the benefit factor which organism actually may get could be in any scale between 1 and 2 in nature. This motivates us for changing benefit factor $\left(\mathrm{BF}_{1}\right)$ to adaptive benefit factor (ABF) which gives good convergence, superior search ability, and harmony between exploration and exploitation:

$X_{\text {inew }}=X_{i}+\operatorname{rand}(0,1) \times\left(X_{\text {best }}-\right.$ Mutual_Vector $\left.\times A B F\right)$.

$X_{\text {jnew }}=X_{j}+\operatorname{rand}(0,1) \times\left(X_{\text {best }}-\right.$ Mutual_Vector $\left.\times \mathrm{BF}_{2}\right)$

where Mutual_Vector is defined as Eq. 3.

$\mathrm{ABF}= \begin{cases}f_{i}\left(X_{i}\right) / f_{i}\left(X_{\text {best }}\right), & \text { if } f_{i}\left(X_{\text {best }}\right) \neq 0 \\ 1+\operatorname{round}[\operatorname{rand}(0,1)], & \text { if } f_{i}\left(X_{\text {best }}\right) \neq 0\end{cases}$

$\mathrm{ABF}= \begin{cases}1, & \text { if } \mathrm{ABF}<1 \\ 2, & \text { if } \mathrm{ABF}>2 \\ \mathrm{ABF}, \text { otherwise }\end{cases}$

The adaptive benefit factor as shown in Eqs. $(10,11)$ is applied for minimization problem. Here value of the design variables in this algorithm may change to a small extent or to a significant extent as they are governed by various factors. The large and small changes in the design variables represent the exploration and exploitation of a search space, respectively. For an accurate optimization of structure with minimization of objective function, a good balance between the exploration and exploitation is needed. Exploration refers to the process of finding promising areas of a search space and leads to global search. Exploitation, however, is the local search around desired solutions found in the exploration phase.

Due implementation of adaptive benefit factor, the term Mutual_Vector $\times$ ABFgives a good balance between exploration and exploitation for the relationship characteristic between organism $X_{i}$ and $X_{j}$ compared with those of initial $\mathrm{BF}_{1}$ and $\mathrm{BF}_{2}$ rand $(0,1)$ values. This leads to good diversity with faster convergence and more stability of $\left(X_{\text {best }}-\right.$ Mutual_Vector $\left.\times \mathrm{ABF}\right)$. As a result, new 
solutions of $X_{\text {inew }}$ and $X_{\text {jnew }}$ lies inside feasible region that minimize the deviation of the objective function between the best organism and the whole ecosystem. Consequently, the stability of the algorithm based on the evaluation of standard deviation increases, and the algorithm demands less numbers of analyses to converge a global optimal solution. Eventually exploration ability of mutualism phase is increase due to implemented modification in Benefit factor.

$\mathrm{ABF}$ leads the algorithm to explore non-visited region in search space when population (' $i$ ' or ' $j$ ') is away from the best population and also helps in increase the convergence rate of solution when population is nearby to best solution. This shows that MSOS with ABF leads to the global optimal solution with a good balance between exploration and exploitation of optimization algorithm.

\subsection{Modification in parasitism phase}

A parasitism phase is important in upgrading the exploration capacity of SOS. However, it is also experienced that over exploration results in higher computational cost. In this phase, a large number of new solutions get rejection due to inferior objective functional values compared to previous one. In parasitism phase of basic SOS algorithm, the exploration rate is poor, as the parasite vector is producing with fusion of design variable with a random generated variable in search space. This only results in improvement of existing result, which enhances the exploitation capability of this algorithm. The main reason for modification is to remove the drawback of low exploitation capability of parasitism phase. Many studies show that the exploitation capability of parasitism phase in SOS algorithm is considerably low as compared to exploratory capability. Increasing the number of $F E$ leads to an increase in the convergence time too.

In addition, many researches show the improvement in efficiency of algorithm with modification in parasitism phase $[45,66]$. Therefore, this phase is improved with the modification of parasitism phase. Here it is tried to improve exploitation potential of parasitism phase with maintaining global optimal solution as well in search space. Therefore, our motive is to set a perfect balance between exploration and exploitation of search algorithm. In this the proposed algorithm, exploration is encourage using mutation strategy. The strategy enhances the diversity of population and solutions as well. The proposed approach allows the algorithm to explore different regions of the search space at the same time, avoid the population concentration in one region, and avoid premature convergence. Equation 12 represents the modification in parasite vector:

$$
\text { Modified Parasitism Vector }=\left\{\begin{array}{lc}
X_{i}^{j} & \text { if } 0.5 \leq \operatorname{rand}(0,1) \\
X_{k}^{j} & \text { otherwise }
\end{array}\right.
$$

Here, the randomly generated organism $X_{k}$ is replaced by parasitism vector when parasitism vector function value is better than host $X_{k}$. This is governed by the condition when a random number in $[0,1]$ has a greater value than a threshold $(0.5 \leq \operatorname{rand}(0,1))$, where threshold value 0.5 is adapted iteratively. Otherwise new organism ' $k$ ' is selected randomly from the population. Here if the improved parasitism vector is fitter than the organism ' $k$ ', parasite will kill organism ' $k$ ' and acquire its place. Finally, if the objective function value for the improved parasitism vector gives minimum value than the previous one, then the parasitism vector takes the new position while eliminating previous organism.

Populations are evolving to a fitter version only if their new fitness dominates their pre-interaction fitness. In this case, the old $X_{i}$ will be replaced immediately by $X_{\text {inew }}$. The $X_{i}$ will be moved into advanced population. Otherwise, $X_{\text {inew }}$ will be added into advanced population for selecting the next generation ecosystem. As such, the proposed algorithm can converge faster while maintaining good diversity. Due to above-mentioned modification in parasitism phase exploitation capability increase with high convergence rate and stability of optimal solution.

It should be noted that in this modification commensalism phase kept remain same as in the original SOS algorithm. In commensalism phase, each organism $X_{\text {inew }}$ and $X_{\text {jnew }}$ is compared with the pre-interaction organism $X_{i}$ and $X_{j}$ to choose a better organism for the next step. Parameter $\left(X_{\text {best }}-X_{j}\right)$, of Eq. (6) echoes the leverage getting by $X_{i}$ due its relationship with $X_{j}$. In this phase, an arbitrary organism $X_{i}$ interacts with randomly selected another organism $X_{j}$ in ecosystem in which only organism $X_{i}$ benefited, while other, i.e., $X_{j}$ remain unaffected. The organism updated only when the function value $F\left(X_{\text {inew }}\right)$ fitter than previous one. This helps $X_{i}$ to ameliorate in ecosystem with respect to existing best organism $X_{\text {best }}$. It is clear that the worse organism in each pair is not selected and convergence speed increased with better organism selection. In this phase exploitation ability and convergence speed are improved due to the decreased search space. Therefore, in this phase a good exploitation near the best organism region is observed in search space which accelerates the convergence of algorithm.

However, with the afore-implemented modifications in the mutualism and parasitism phases of MSOS, the exploration and exploitation abilities are balanced. Moreover, the suggested parameter values in the MSOS have faster convergence speed against the original SOS. It can be concluded that MSOS significantly improves the computational cost and the convergence of the original SOS, but still attaining the global optimal solution with high accuracy and reliability. Both implementations aim at increasing the exploration and exploitation capability and thus enhancing the convergence speed with higher precision, 


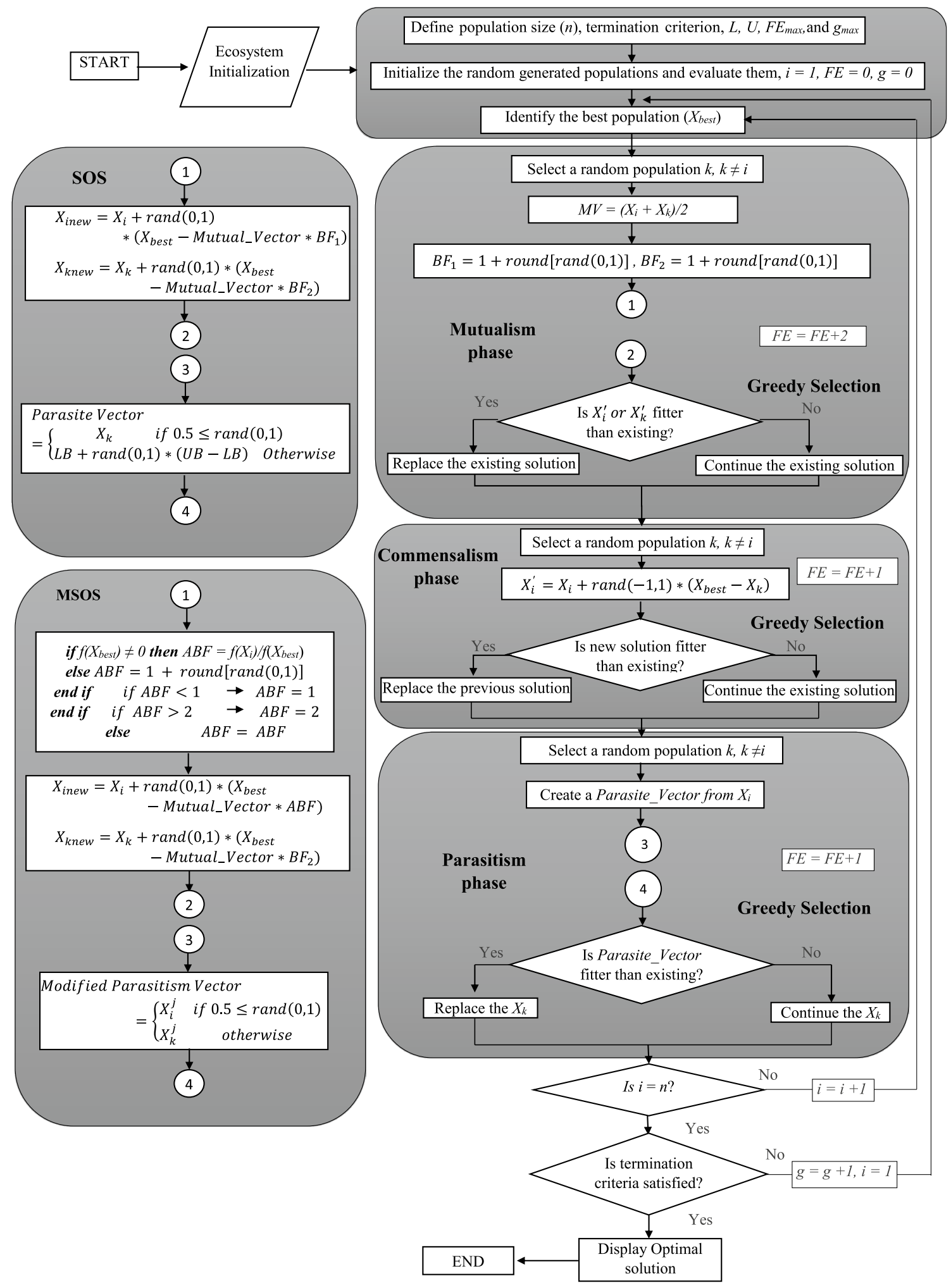

Fig. 1 Flowchart of the SOS and MSOS algorithms 
and stronger robustness than basic SOS. From above modification, a balanced trade-off between the exploration and exploitation abilities is, therefore, achieved. Modification increases the diversity of the population and improves the ability of the algorithm to explore and exploit, as well as preventing the algorithm from premature convergence. Furthermore, the proposed algorithm is simple and easy to implement.

The proposed algorithms work in three phases: the mutualism phase, the commensalism phase, and the parasitism phase. In addition, each phase is governed by a number of generations and various factors. A schematic diagram of MSOS and SOS is presented in Fig. 1, which gives various steps of these algorithms like initialization, mutualism phase, commensalism phase, parasitism phase, and termination criteria. The subsequent sections examine the efficiency of MSOS with respect to the shape and sizing problems.

\section{The formulation of the design problem}

The basic objective of structural optimization problem is to minimize the mass of the truss via finding optimal element cross section area and optimal nodal positions subjected to multiple frequency constraints. Therefore, the objective function is the mass of truss by neglecting lumped mass at nodes

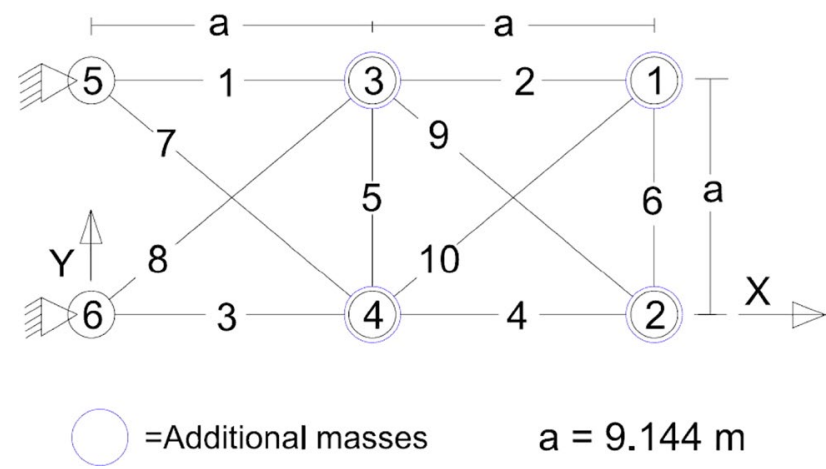

while keeping nodal coordinates and elemental cross-section areas as design variables.

The mathematical formulation corresponding to the problem considered in this work is as follows:

Find, $X=\{A, N\}$, where $A=\left\{A_{1}, A_{2}, \ldots, A_{m}\right\}$ and

$$
N=\left\{N_{1}, N_{2}, \ldots, N_{n}\right\}
$$

to minimize, Mass of truss,

$F(X)=\sum_{i=1}^{m} A_{i} \rho_{i} L_{i}$

Subjected to

$g_{1}(X): f_{q}-f_{q}^{\min } \geq 0$

$g_{2}(X): f_{r}-f_{r}^{\max } \leq 0$

$g_{3}(X): A_{i}^{\min } \leq A_{i} \leq A_{i}^{\max }$

$g_{4}(X): N_{j}^{\min } \leq N_{j} \leq N_{j}^{\max }$

where $i=1,2, \ldots, m ; j=1,2, \ldots, n$

where $A_{i,} \rho_{i}$, and $L_{i}$ signify the cross-sectional area, material density, and length of the bar ' $i$ ', respectively. $N_{j}$ presents nodal coordinate $\left(x_{j}, y_{j}, z_{j}\right)$ of ' $j$ th' node. $f_{q}$ and $f_{r}$ are ' $q$ th' and ' $r$ th' natural frequencies, respectively. The superscripts, 'max' and 'min' signify the maximum and minimum allowable limits, respectively. The finite element method is applied as analyzer to calculate fundamental Eigen values and natural frequencies of the truss structure.

Solving constrained optimization problem at times become infeasible as the solutions at times get stuck in the local optima, especially in the problems having disjoint search space. Under these scenarios Penalty function methods are used which convert a constrained problem into an unconstrained one, where the 'Penalty Function' penalizes the infeasible solutions to move toward desirable feasible solutions.

Fig. 2 10-bar truss

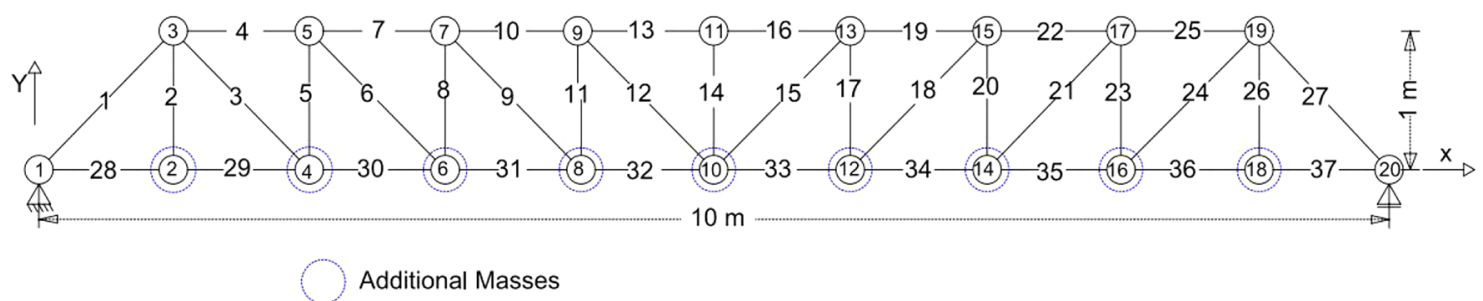

Fig. 3 37-bar truss 


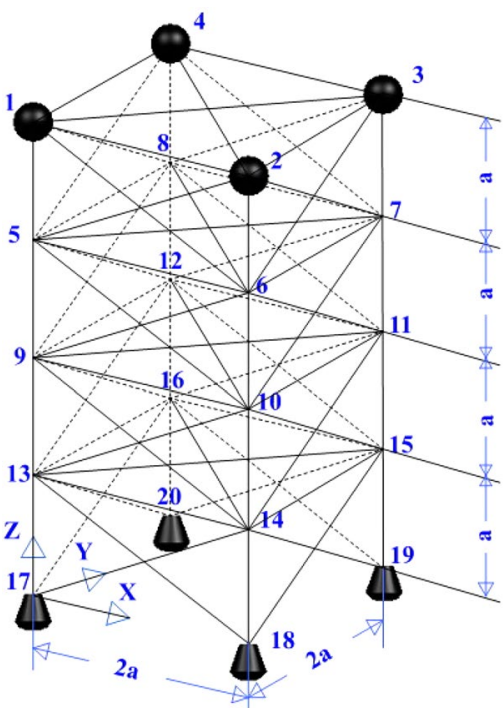

$=$ Addition
$a=1.5240$

Fig. 4 72-bar truss
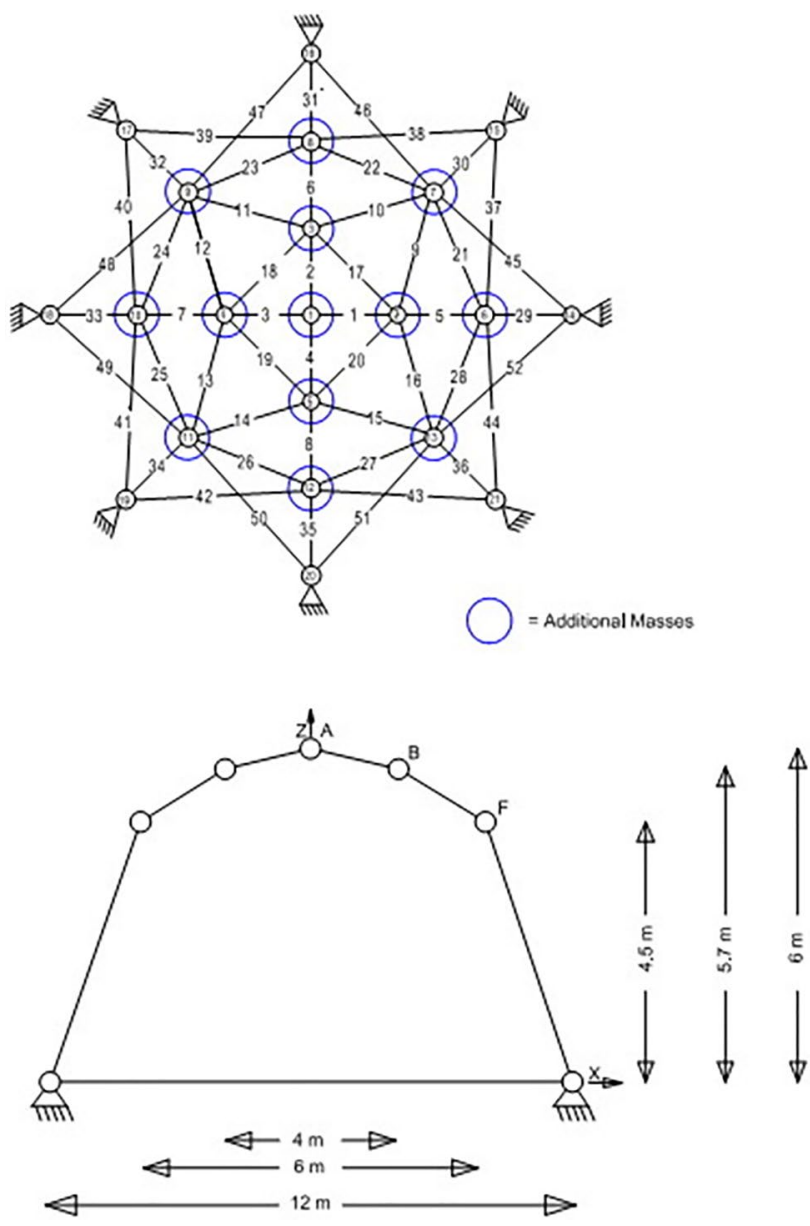

Fig. 5 52-bar truss
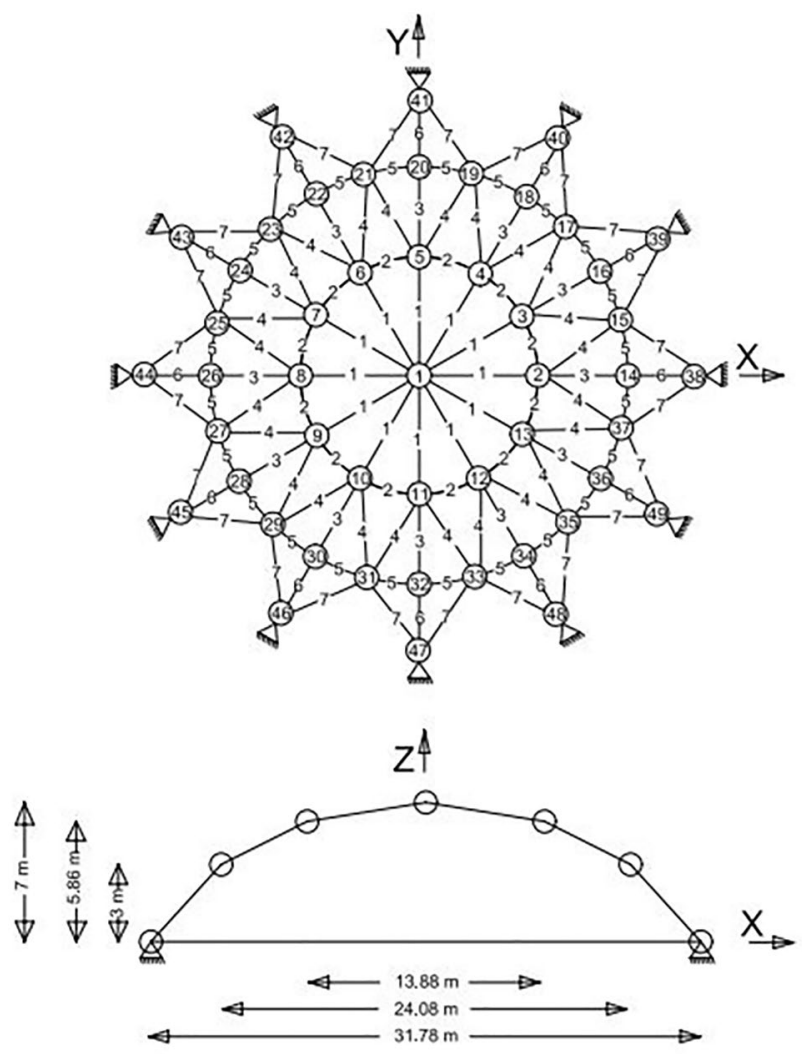

Fig. 6 120-bar truss

The objective function is penalized to handle frequency limits. There is no penalty for non-violation of the limits; otherwise, the penalty function is considered as follows [28]:

Penalized $F(X)=\left\{\begin{array}{l}F(X), \text { if no violation of limits } \\ F(X) \times F_{\text {penalty }}, \text { otherwise }\end{array}\right.$

$F_{\text {penalty }}=\left(1+\varepsilon_{1} \times \complement\right)^{\varepsilon_{2}}, \complement=\sum\left(\complement_{q}+\complement_{r}\right)$,

$\complement_{q}=\left|1-\frac{\left|f_{q}-f_{q}^{\min }\right|}{f_{q}^{\min }}\right|, \complement_{r}=\left|1-\frac{\left|f_{r}-f_{r}^{\max }\right|}{f_{r}^{\max }}\right|$

The parameters $\varepsilon_{1}$ and $\varepsilon_{2}$ are selected by considering their nature. In this investigation, values of $\varepsilon_{1}$ and $\varepsilon_{2}$ are set as 3 as per previous studies $[48,75]$.

\section{Truss problems and discussions}

In this section, the proposed algorithm is tested on six challenging benchmark trusses (as shown in Figs. 2, 3, 4, 5, 6, and 7). Here the consideration is to perform size and shape optimization of given trusses under multiple natural frequency limits. Later on, soundness and viability of the proposed 
Fig. 7 200-bar truss

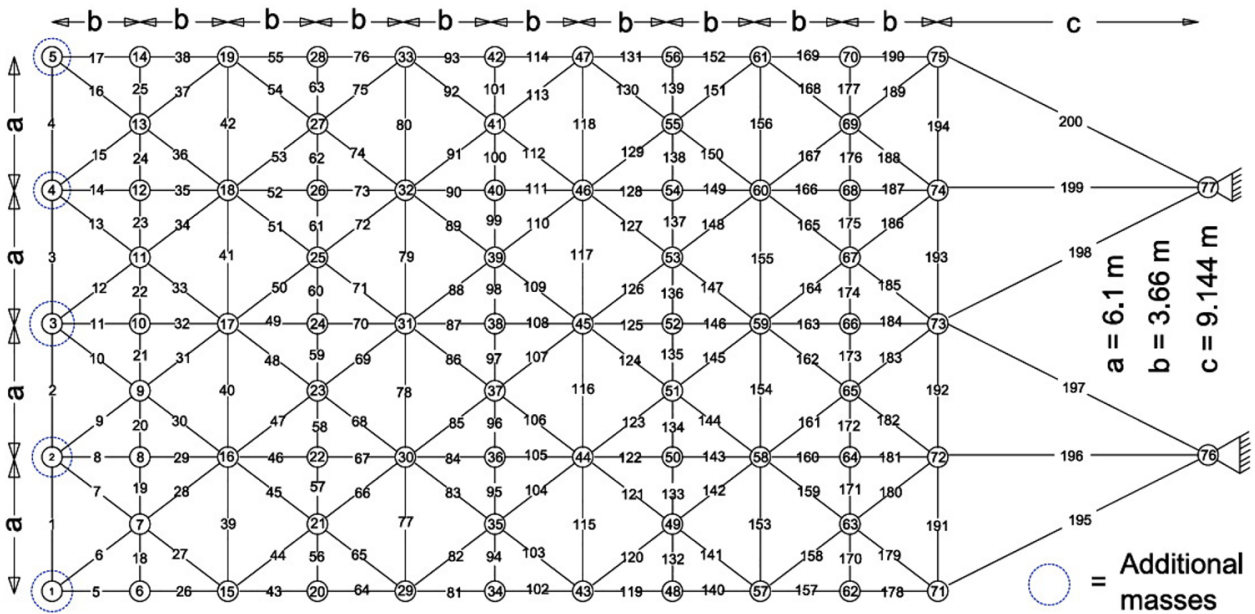

Table 1 Design parameters of the benchmark trusses

\begin{tabular}{|c|c|c|c|c|c|c|}
\hline & The 10-bar truss & The 37-bar truss & The 72-bar truss & The 52-bar truss & The 120 -bar truss & The 200-bar truss \\
\hline Design variables & $A_{i}, i=1,2, \ldots, 10$ & $\begin{array}{l}A_{i}, i=1,2, \ldots, 14 \\
y_{j}, j=3,5,7,9,11\end{array}$ & $G_{i}, i=1,2, \ldots, 16$ & $\begin{array}{l}G_{i}, i=1,2, \ldots, 8 \\
z_{A}, z_{B}, z_{F}, x_{B}, x_{F}\end{array}$ & $G_{i}, i=1,2, \ldots, 7$ & $G_{i}, i=1,2, \ldots, 29$ \\
\hline Limits $f(H z)$ & $\begin{array}{l}f_{1} \geq 7 \\
f_{2} \geq 15 \\
f_{3} \geq 20\end{array}$ & $\begin{array}{l}f_{1} \geq 20 \\
f_{2} \geq 40 \\
f_{3} \geq 60\end{array}$ & $\begin{array}{l}f_{1} \geq 4 \\
f_{3} \geq 6\end{array}$ & $\begin{array}{l}f_{1} \leq 15.916 \\
f_{2} \geq 28.648\end{array}$ & $\begin{array}{l}f_{1} \geq 9 \\
f_{2} \geq 11\end{array}$ & $\begin{array}{l}f_{1} \geq 5 \\
f_{2} \geq 10 \\
f_{3} \geq 15\end{array}$ \\
\hline Size variables, $A_{i}\left(\mathrm{~cm}^{2}\right)$ & {$[0.645,50]$} & {$[1,10]$} & {$[0.645,30]$} & {$[1,10]$} & {$[1,129.3]$} & {$[0.1,30]$} \\
\hline Shape variables & - & $y_{j} \in[0.1,3] \mathrm{m}$ & - & $\pm 2 \mathrm{~m}$ & - & - \\
\hline $\begin{array}{l}\text { Material den- } \\
\quad \text { sity } \rho\left(\mathrm{kg} / \mathrm{m}^{3}\right)\end{array}$ & 2770 & 7800 & 2770 & 7800 & 7971.81 & 7860 \\
\hline $\begin{array}{l}\text { Young modulus } \\
E(P a)\end{array}$ & $6.98 \times 10^{10}$ & $2.1 \times 10^{11}$ & $6.98 \times 10^{10}$ & $2.1 \times 10^{11}$ & $2.1 \times 10^{11} \mathrm{~Pa}$ & $2.1 \times 10^{11}$ \\
\hline
\end{tabular}

MSOS algorithm was evaluated for different continuous section truss design. Furthermore, the results are compared with previous results obtained through various existing MHs like FA, TLBO, OC, GA, CSS, CBO, TWO, SOS, and VPS.

\subsection{The 10-bar truss}

The first benchmark problem of 10-bar truss, shown in Fig. 2, is considered here to investigate the effect of various design parameters (given in Table 1). This problem results already been examined by various researchers is shown in Table 2. For given truss problem, ten continuous design variables are considered for sizing function and at all free nodes (nodes 1-4) of the given truss a $454.0 \mathrm{~kg}$ lumped mass is added, as shown in Fig. 2.

MSOS is tested check effects on size optimization by assuming population size as 20 and maximum Function Evaluations as 4000. Table 2 illustrates design variables, truss masses, Standard Deviation (SD) of mass, FE, and frequency responses obtained for 100 independent runs. The result table presents that MSOS and SOS find the minimum mass 524.5747 and $525.2789 \mathrm{~kg}$, respectively. In addition, MSOS finds the best solution as compared to similar results detailed in literature. Therefore, the results of MSOS are compared with the results of the other MHs. The mass saving for MSOS is $18.18,13.41,10.57,7.38,4.68,10.42,6.71$, $4.52,11.16,7.66,0.70,0.35,0.25,0.70$, and $0.16 \mathrm{~kg}$ as compared to those obtained from NHGA, PSO, NHPGA, CSS, enhanced CSS, HS, FA, CSS-BBBC, hybrid OC-GA, TWO, SOS, SOS-ABF1, SOS-ABF2, SOS-ABF1\&2, and ISOS, respectively.

Mean mass (Avg.) obtained using MSOS and SOS is 527.7970 and $531.4033 \mathrm{~kg}$, respectively. As per results, MSOS presents the best Avg. solution among the considered MHs. It is also observed that SOS and its variants find better Avg. solution as compared to similar solutions presented in literature. The Avg. mass saving for MSOS is 13.09 , 8.59, $10.73,9.88,7.27,7.75,3.61,0.83,0.75,0.91$, and $2.23 \mathrm{~kg}$ as compared to those obtained from PSO, CSS, enhanced CSS, HS, FA, TWO, SOS, SOS-ABF1, SOS-ABF2, SOS$\mathrm{ABF} 1 \& 2$, and ISOS, respectively. 


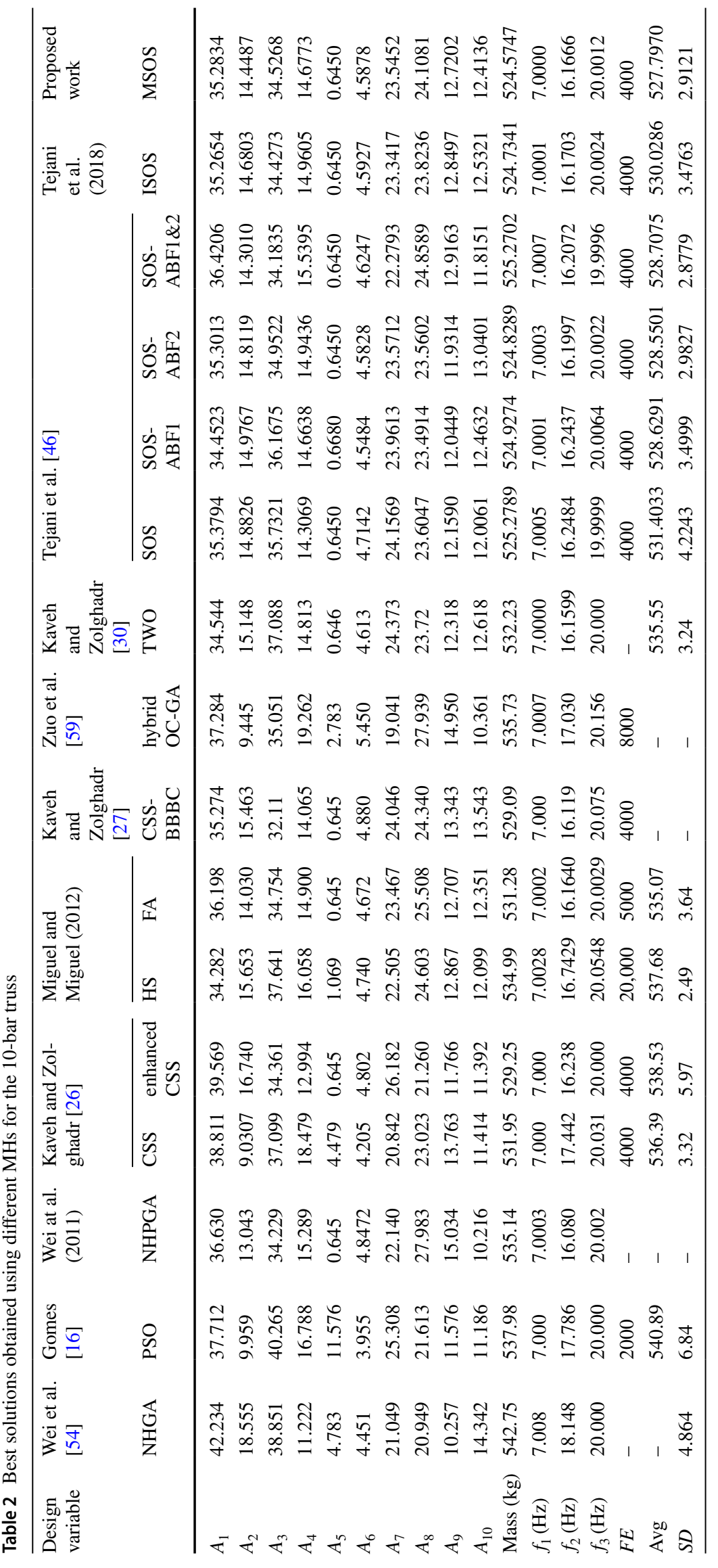




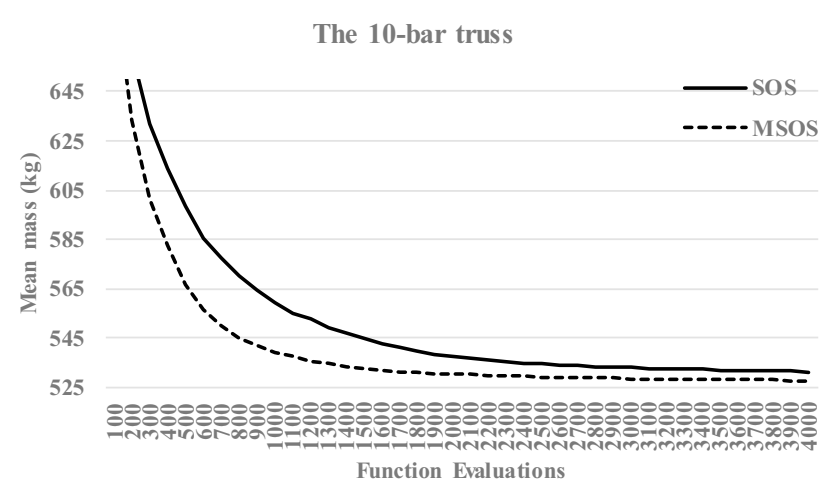

Fig. 8 Convergence curve of the 10-bar truss

MSOS and SOS find SD as 2.9121 and $4.2243 \mathrm{~kg}$, respectively. It is evident that MSOS finds better solution as SD compare to SOS and other MHs except HS and SOSABF1\&2. It should be noted that maximum $F E$ used in MSOS and SOS is fairly small as compared to HS, FA, and hybrid OC-GA. This valuation specifies that the solutions obtained using MSOS and SOS are more reliable and superior with the other solutions stated in literature. However, few studies are available in the literature which demonstrate competitive edge to proposed algorithm like VPS which gives Avg. and SD values of 535.64 and $2.55 \mathrm{~kg}$, respectively, after 4620 analyses, a novel adaptive hybrid evolutionary firefly algorithm (AHEFA) suggested by Lieu et al. [88] which gives Avg. and SD values of 525.1623 and $1.9155 \mathrm{~kg}$, respectively, after 5860 function analyses, Roulette wheel selection-Elitist-Differential Evolution (ReDE) recommended by Ho-Huu et al. [89] results into Avg. and SD values of 525.7039 and $1.3415 \mathrm{~kg}$, respectively, after 20,000 average function analyses. Kaveh and Javadi [90] suggested an efficient hybrid algorithm HRPSO (which compound particle swarm, ray optimizer, and harmony search strategy) which gives Avg. and SD values of 524.88 and $2.253 \mathrm{~kg}$, respectively, after 10 independent runs. Similarly, cyclical parthenogenesis algorithm (CPA) proposed by Kaveh and Zolghadr (2016) gives Avg. and SD values of 533.66 and $2.67 \mathrm{~kg}$, respectively, after 12,800 structural analyses.

Figure 8 shows a convergence graph of mean mass with respect to FE for MSOS and SOS. The mean mass is computed by considering the average mass of all runs for each generation. The graph indicates that MSOS converges faster in nearly $2000 \mathrm{FE}$. In addition, it is identified that MSOS outperforms the basic SOS.

\subsection{The 37-bar truss}

The second benchmark problem considered here is shown in Fig. 3, which is a 37-bar truss, simply supported bridge. Initially it was considered by Wang et al. [53] and later many researchers investigate this truss problem (as shown in Table 3). Various design parameters considered here is depicted in Table 1. In addition, all free nodes here considered having a lumped mass of $10 \mathrm{~kg}$ attached to the lower chord of truss. Moreover, lower cords of given truss problem are assumed to have fixed rectangular cross-section of $0.4 \mathrm{~cm}^{2}$, whereas the remaining bars are clustered into fourteen groups by considering symmetry of structure about its middle vertical plane. Here lower nodes are considered to be fixed and upper nodes have a possibility of shifting vertically as structural symmetry has been considered along vertical plane. Due to above consideration this problem has five shape and fourteen size variables.

MSOS and SOS are investigated by assuming population size as 20 and $F E$ as 4000 . The solutions are presented in Table 3. MSOS and SOS find the best solution as 360.3018 and $360.8658 \mathrm{~kg}$, respectively. The results show that SOS and its variants find nearly similar and better solutions with similar results reported in literature. However, maximum FE used in SOS and its variants is very small as compared to PSO, HS, and FA.

MSOS and SOS present Avg. solutions as 362.9610 and $364.8521 \mathrm{~kg}$, respectively. MSOS and SOS present SD of mass as 1.7265 and $4.2278 \mathrm{~kg}$, respectively. It observed from the solutions that that MSOS archives better solution as Avg. and SD of mass among the considered MHs for $4000 \mathrm{FE}$. Meanwhile, many competitive solutions are also available in literature like a new Particle Swarm Ray Optimization (PSRO) proposed by Kaveh and Zolghadr [92] which gives the result of mean weight and standard deviation after 20 independent runs as 362.65 and $1.30 \mathrm{~kg}$, respectively. Similarly, CPA gives Avg. and SD values of 360.93 and $0.65 \mathrm{~kg}$, respectively, after 12,800 structural analyses, VPS gives Avg. and SD values of 360.23 and $0.22 \mathrm{~kg}$, respectively, after 7940 structural analyses. In addition, CBO gives the solution of Avg. $360.4463 \mathrm{~kg}$ and SD of $0.35655 \mathrm{~kg}$ after 6000 number of analysis. Moreover, ReDE results into Avg. and SD values of 359.9944 and $0.1493 \mathrm{~kg}$, respectively, after 12,579 average function analyses.

Figure 9 shows the convergence graph of the 37-bar truss. Graph is plotted between mean mass vs. FE for the proposed algorithms. The convergence graph indicates that MSOS converge faster and achieves good optimal results as compared to SOS. Moreover, MSOS shows early convergence nearly within $3000 \mathrm{FE}$. In addition, it is identified that from the obtained results, MSOS performs better compare to basic SOS. Therefore, the performance of the proposed modified algorithm is better as compared to its basic version in terms of statistical results and convergence. 


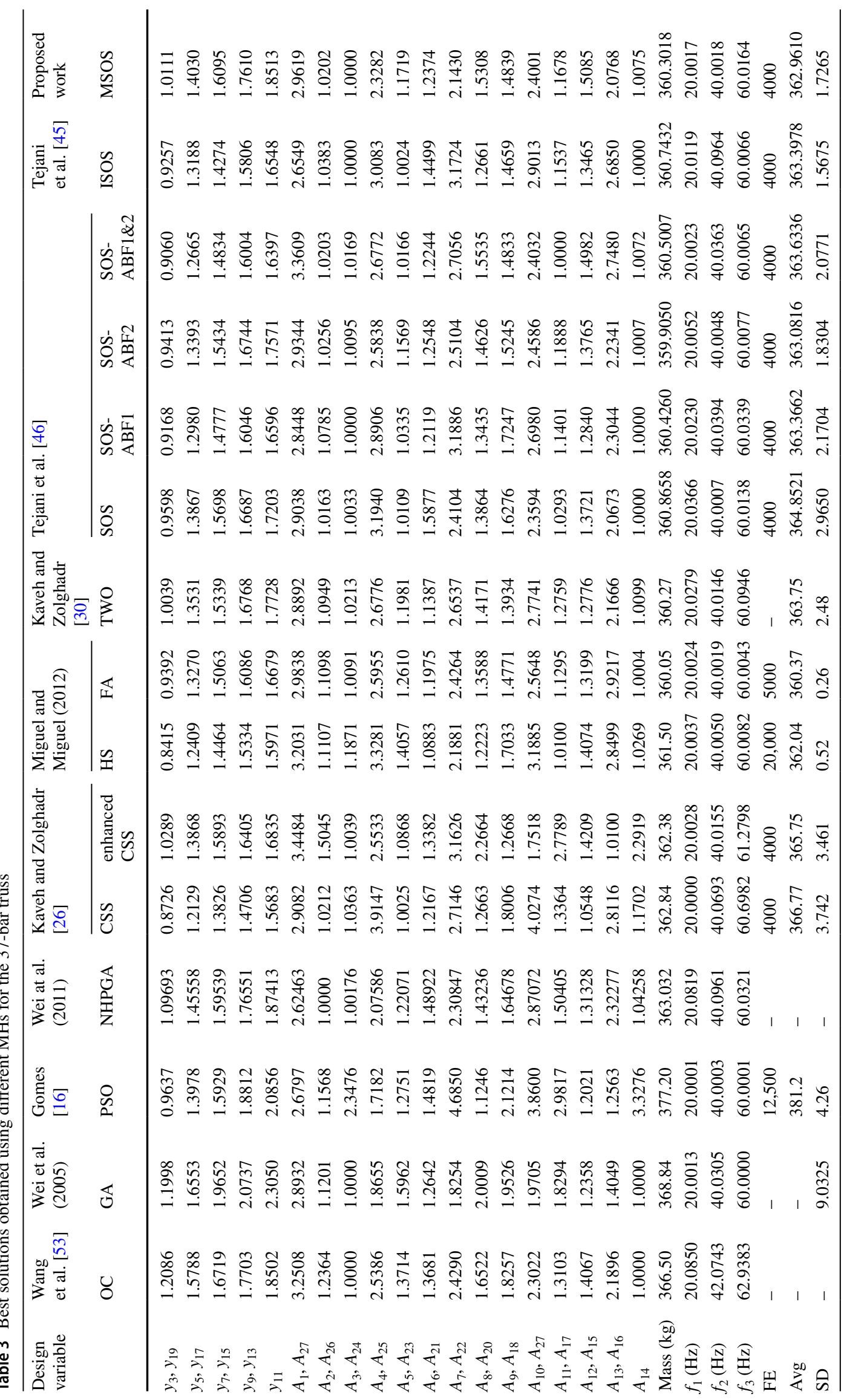




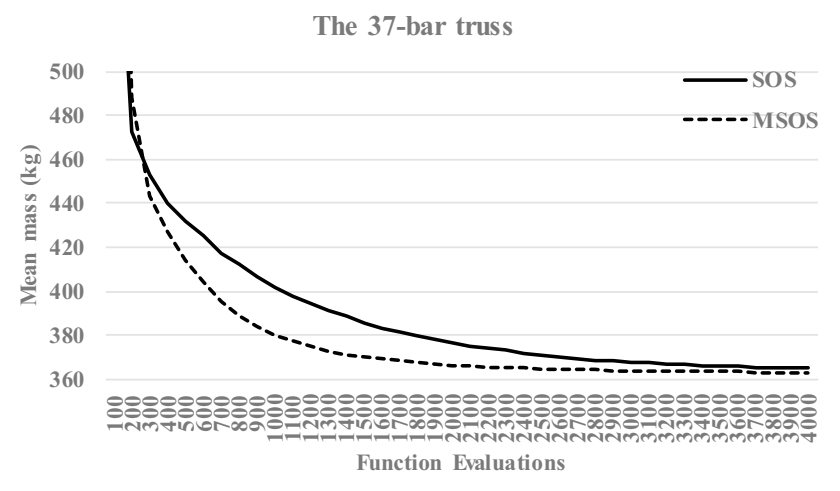

Fig. 9 Convergence curve of the 37-bar truss

\subsection{The 72-bar truss}

The third benchmark truss problem taken here is manifested in Fig. 4. Similar to earlier cases this truss problem also has been investigated by many researchers at large scale as sizing problem. Table 1 represents the deign consideration for this problem. Here due to assumption of vertical plane symmetry of structure similar to previous cases, sixteen groups of bars are considered. Furthermore, at all top position nodes (nodes 1-4) a lumped mass of $2770 \mathrm{~kg}$ is assumed to be attached, as shown in Fig. 4.

MSOS and SOS are investigated by assuming population size as 20 and $F E$ as 4000 . The solutions are presented in Table 4. The best solution found using MSOS and SOS is 324.346 and $325.5585 \mathrm{~kg}$, respectively. It is observed from solutions that MSOS performs better compared to SOS and its other variants and similar solutions reported in literature. Moreover, MSOS performs better among considered MHs. Therefore, results obtained using MSOS are compared with the results of the other MHs mass saving for MSOS is $4.47,4.05,3.16,0.41,3.22,3.23,4.48,1.21,0.74,0.34$, 0.89 , and $0.72 \mathrm{~kg}$ compared to those obtained from CSS, enhanced CSS, CSS-BBBC, CBO, TLBO, MC-TLBO, TWO, SOS, SOS-ABF1, SOS-ABF2, SOS-ABF1\&2, and ISOS, respectively.

The results signify that MSOS and SOS find Avg. solution as 326.7847 and $331.1228 \mathrm{~kg}$, respectively. MSOS and SOS find SD of mass as 2.1968 and $4.2278 \mathrm{~kg}$, respectively. It can be seen from the results that MSOS finds best result as Avg. and SD of solutions among the considered MHs with 4000 FEs. It observed that maximum FE consumed by CBO, TLBO, and MC-TLBO is significantly better as compared to the other MHs. However, few studies are available in the literature which illustrate competitive solutions than proposed algorithm like CPA gives Avg. and SD values of 330.91 and $1.84 \mathrm{~kg}$, respectively, after 12,800 structural analyses, VPS gives Avg. and SD values of 327.670 and $0.018 \mathrm{~kg}$, respectively, after 4720 structural analyses, HRPSO gives Avg. and
SD values of 324.497(in lb.) and $3.948 \mathrm{~kg}$, respectively, after 10 independent runs. Similarly, ReDE results into Avg. and SD values of 324.3219 and $0.0516 \mathrm{~kg}$, respectively, after 11,116 average function analyses, AHEFA gives Avg. and SD values of 324.4109 and $0.2420 \mathrm{~kg}$, respectively, after 8860 function analyses.

Figure 10 illustrates a convergence graph of the mean mass for MSOS and SOS. As observed from the graph, the mean mass for $4000 \mathrm{FE}$ is $326.7847 \mathrm{~kg}$ and $331.1228 \mathrm{~kg}$ for MSOS and SOS, respectively. It can be seen from the convergence graph that MSOS converges better than SOS. This study specifies that the solutions obtained using MSOS are more reliable and proficient as compared to similar solutions obtained using other MHs.

\subsection{The 52-bar truss}

The fourth problem selected, as shown in Fig. 5, is 52-bar dome shape truss problem. This truss problem was first studied by Lin et al. (1982) for shape and sizing optimization and later it was considered by several other scholars, as shown in Table 5. For this problem various design parameters consideration are illustrated in Table 1. As in first case, here a $50 \mathrm{~kg}$ lumped mass is assumed to be attached at free nodes. The elements of structure are clustered into eight groups considering $z$-axis symmetry and also to keep dome symmetric free nodes are allowed to shift $\pm 2 \mathrm{~m}$ in each direction of the vertical plane.

MSOS and SOS are investigated by assuming population size as 20 and FE as 4000. Table 5 illustrates the solutions obtained using MSOS, SOS, and other MHs stated in literature. The solutions show that MSOS and SOS propose trusses with the best mass of 193.773 and $195.4969 \mathrm{~kg}$, respectively. It is identified that MSOS ranks first, whereas ISOS ranks second among the considered MHs. The mass saving for MSOS is 104.23, 42.27, 34.61, 11.46, 3.56, 21.17, $3.76,3.54,1.58,0.48,1.72,1.04,1.40,4.49$, and $0.98 \mathrm{~kg}$ compared to those obtained from Bi-factor algorithm, NGHA, PSO, CSS, enhanced CSS, HS, FA, CSS-BBBC, DPSO, TWO, SOS, SOS-ABF1, SOS-ABF2, SOS-ABF1\&2, and ISOS, respectively.

The results signify that MSOS and SOS present Avg. solutions as 204.4279 and $214.6676 \mathrm{~kg}$, respectively. The results indicate that MSOS finds better Avg. solutions as compared to other MHs stated in literature except DPSO. In addition, the maximum $\mathrm{FE}$ consumed in the proposed MHs is fairly small as compared to PSO, HS, FA, and DPSO. MSOS and SOS find SD as 8.4014 and $15.1499 \mathrm{~kg}$, respectively. However, few studies are also available in the literature which exhibit competitive solutions than proposed algorithm like CPA gives Avg. and SD values of 198.81 and $3.71 \mathrm{~kg}$, respectively, after 12,800 structural analyses, HRPSO gives Avg. and SD values of 193.361 and $17.637 \mathrm{~kg}$, 


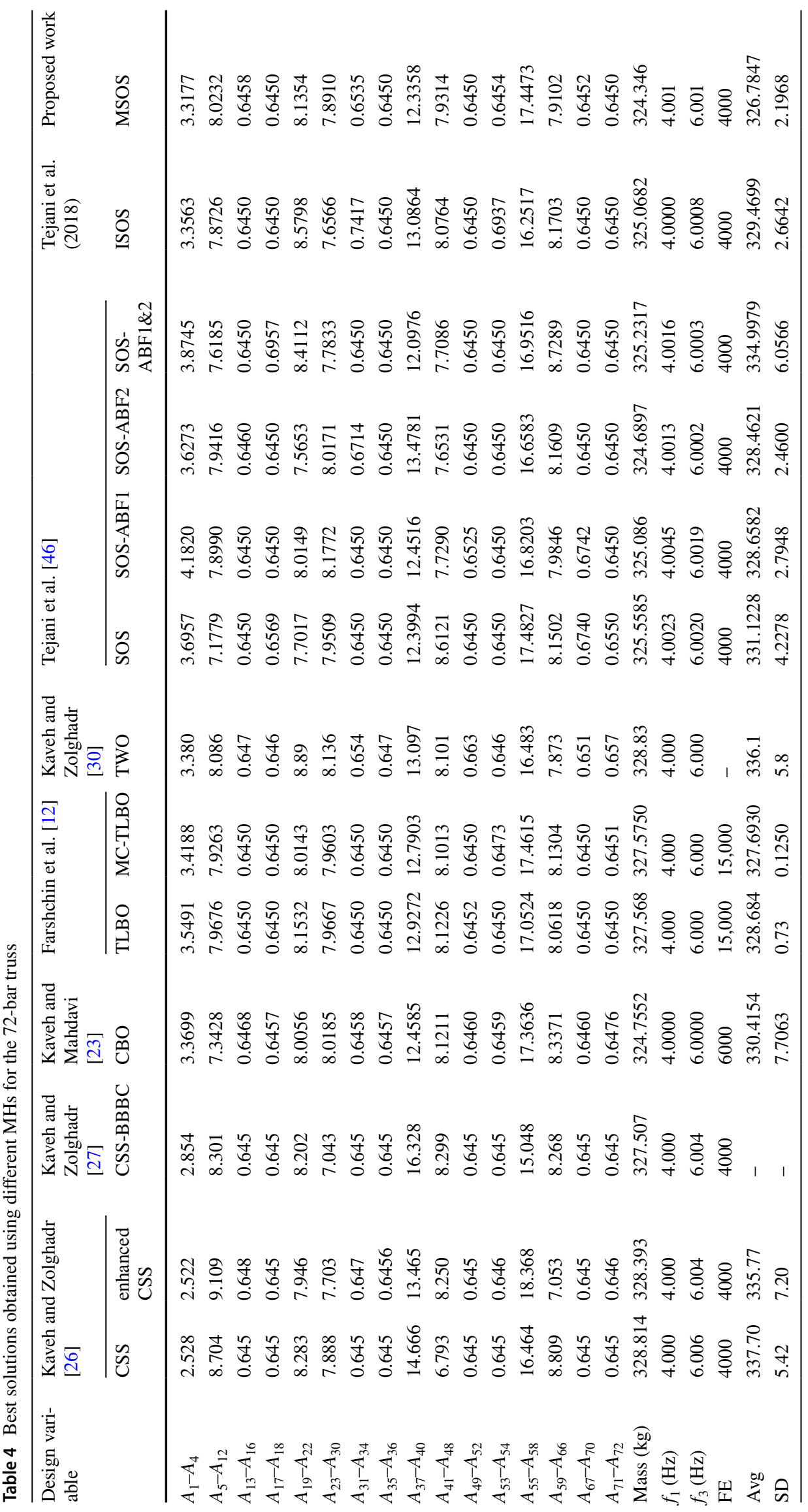




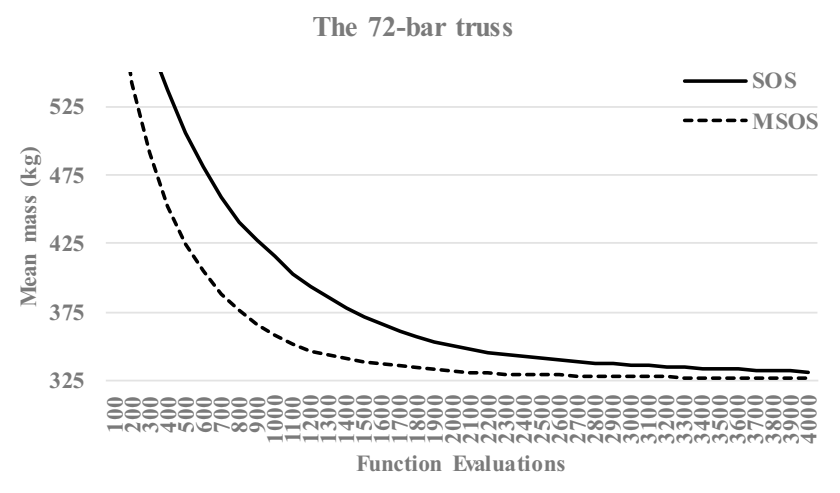

Fig. 10 Convergence curve of the 72-bar truss

respectively, after 10 independent runs, AHEFA gives Avg. and SD values of 198.7290 and $4.4108 \mathrm{~kg}$, respectively, after 12,120 function analyses. Similarly, ReDE results into Avg. and SD values of 195.4260 and $3.8587 \mathrm{~kg}$, respectively, after 14,749 average function analyses. In addition, an Improved ray optimization (IRO) Kaveh et al. [82] gives Avg. and SD values of 196.43 and $1.81 \mathrm{~kg}$, respectively, after 17,000 structural analyses.

It is observed from the solutions that that MSOS gives better Avg. solution and SD as compared to basic SOS. Figure 11 compares the convergence characteristic curve of the mean mass for MSOS and SOS. The convergence graph indicates that MSOS converges faster and achieves good optimal results as compared to SOS. Moreover, MSOS shows early convergence nearly within $3000 \mathrm{FE}$. This study indicates that the results of MSOS, SOS, and its other variants are more reliable and proficient as compared to the results of the other considered MHs. In addition, MSOS performs more efficiently as compared to basic SOS.

\subsection{The 120-bar truss}

The fifth benchmarks problem considered here is 120 bar truss problem, as shown in Fig. 6.This truss has a 3-D dome structure which was first considered by Kaveh and Zolghadr [27] for size optimization. Again, for this truss problem the design parameters considered is shown in Table 1. Conversely rather than using single lumped mass as done in previous cases, here a lumped mass of $3000 \mathrm{~kg}$ is added at node1, from 2 to 13 nodes $500 \mathrm{~kg}$ and for rest of free nodes a $100 \mathrm{~kg}$ mass is added. Again, based on the $z$-axis symmetry assumption the elements are clustered into seven group here.

MSOS and SOS are investigated by assuming population size as 20 and FE as 4000. Table 6 illustrates the obtained solutions using SOS and other MHs. It shows that MSOS and SOS find the truss problems with the best mass of 8708.3180 and $8713.3030 \mathrm{~kg}$, respectively. The results show that SOS, MSOS, and its other variants give better solutions compared to similar solutions detailed in literature. In addition, MSOS ranks first among the considered MHs. MSOS gives mass saving of 496.19, 338.02, 180.81, 463.61, $182.16,4.99,3.79,2.01,8.63$, and $1.74 \mathrm{~kg}$ compared to solutions obtained from CSS, CSS-BBBC, CBO, PSO, DPSO, SOS, SOS-ABF1, SOS-ABF2, SOS-ABF1\&2, and ISOS, respectively.

The Avg. solution for MSOS and SOS are 8720.9734 and $8735.3452 \mathrm{~kg}$, respectively. It is observed that MSOS finds better Avg. solution among the considered MHs. The Avg. mass benefit for MSOS is 170.28, 530.87, 175.02, 14.37, $6.45,4.33,69.72$, and $7.62 \mathrm{~kg}$ as compared to those obtained from CBO, PSO, DPSO, SOS, SOS-ABF1, SOS-ABF2, SOS-ABF1\&2, and ISOS, respectively. It is observed evidently that MSOS gives better Avg. solution as compared to similar solutions presented in literature. However, few studies are available in the literature which demonstrate competitive solutions than proposed algorithm like VPS gives Avg. and SD values of 8896.04 and $6.65 \mathrm{~kg}$, respectively, after 6860 structural analyses. Similarly, ReDE results into Avg. and SD values of 8707.5233 and $0.1543 \mathrm{~kg}$, respectively, after 5380 average function analyses, AHEFA gives Avg. and SD values of 8707.5580 and $0.2535 \mathrm{~kg}$, respectively, after 3560 function analyses, IRO gives Avg. and SD values of 8905.21 and $4.92 \mathrm{~kg}$, respectively, after 16,300 structural analyses. In addition, Jalili and Talatahari (2018) proposed a hybrid Charged System Search with Migration-based Local Search algorithm (CSS-MBLS) which gives Avg. and SD values of 8715.60 and $4.95 \mathrm{~kg}$, respectively, after 2400 structural analyses. Furthermore, CBO gives the solution of Avg. 8,891.2540 and SD of $1.7926 \mathrm{~kg}$ after 6000 number of analysis.

MSOS and SOS present SD as 11.7324 and 17.9011, respectively. CBO and DPSO rank first and second, respectively, in terms of SD but maximum FE consumed by these MHs is 50\% higher compare to SOS and its variants. MSOS and SOS-ABF2 are nearly similar in SD and perform better compare to others with 4000 FEs. Figure 12 displays convergence of the mean solutions obtained for MSOS and SOS. It is observed from the convergence graph of MSOS that the objective function converges rapidly within initial 2500 FEs. Convergence graph also indicates that MSOS converges better than SOS and converges to better solutions. This study signifies that the solution obtained using MSOS are more reliable and proficient as compared to SOS and other solutions presented in literature without violation of constraints. 


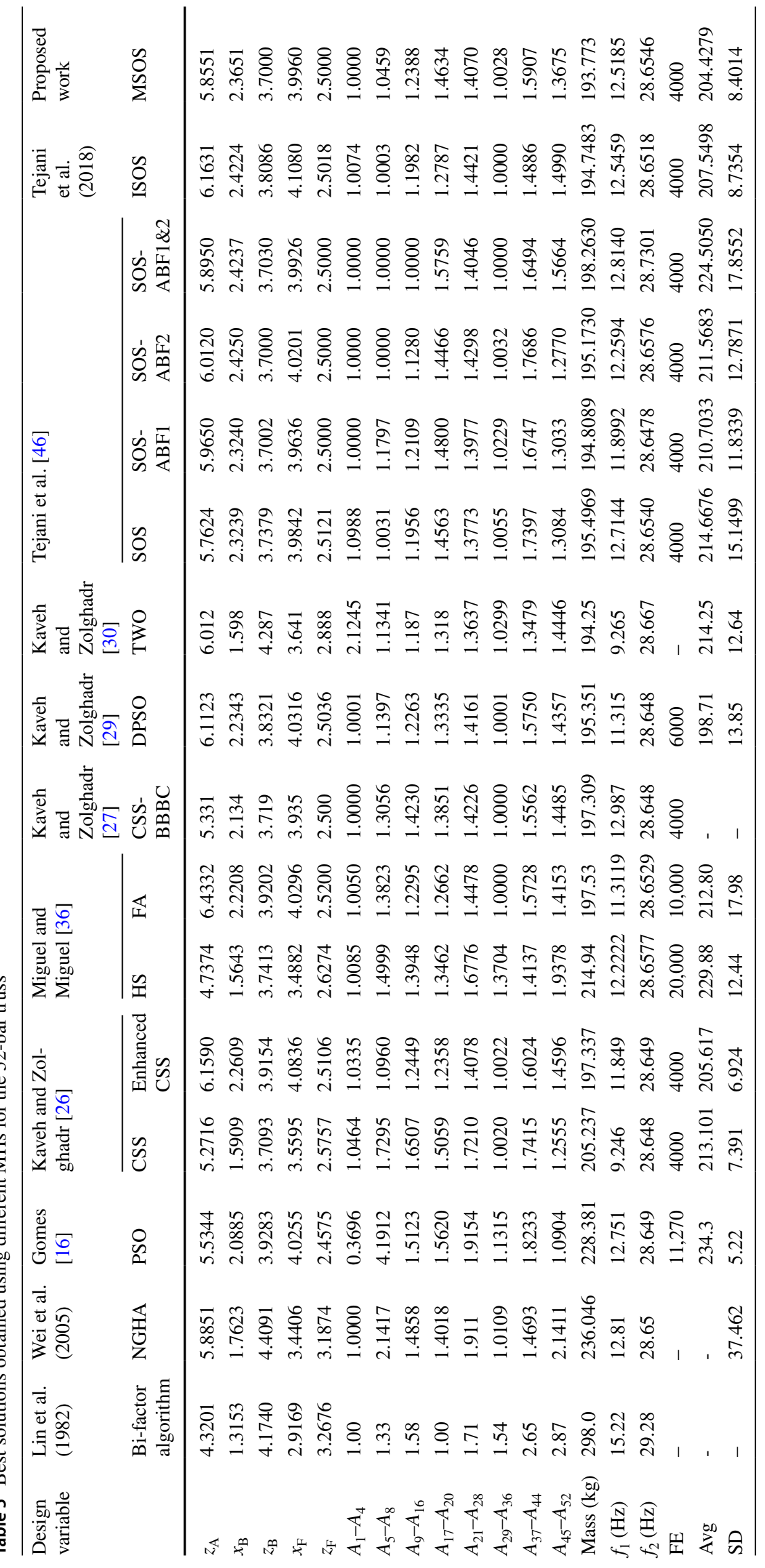




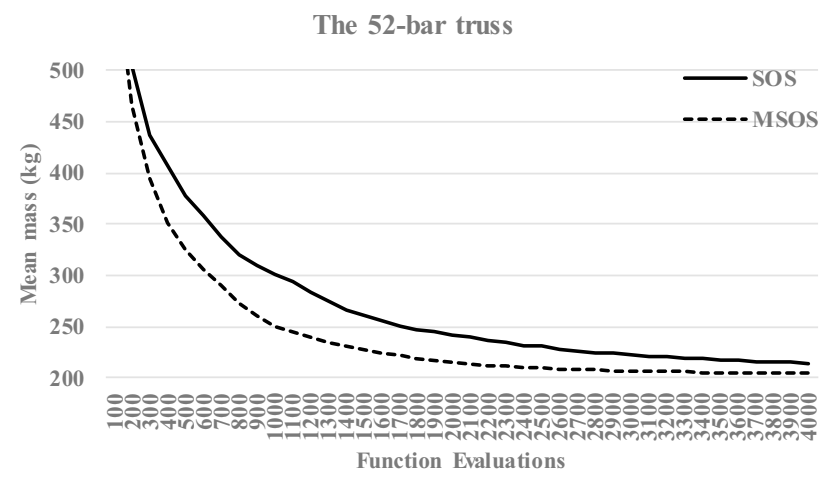

Fig. 11 Convergence curve of the 52-bar truss

\subsection{The 200-bar truss}

Figure 7 illustrates the sixth benchmark problem, i.e., 200 bar truss optimization problem which is a large-scale size optimization problem. Design parameters like design variables, limits, frequency constraints etc. are presented in Table 1. Here a lumped mass of $100 \mathrm{~kg}$ is assumed at all top nodes (nodes 1-5) of structure. Again, based on the $z$-axis symmetry assumption, all the elements are clustered into 29 groups here.

SOS and its variants are assumed with population size of 20 and $F E$ of 10,000. Table 7 illustrates the comparative solutions. The best solutions for MSOS and SOS are 2164.47 and $2180.321 \mathrm{~kg}$, respectively. The results show that MSOS gives better solutions compared SOS and its variants and similar results reported in literature. The solutions show that MSOS finds mass saving of $95.39,134.14$, $38.74,24.61,15.85,0.41,1.33,43.42$, and $4.99 \mathrm{~kg}$ as compared to those obtained from CSS, CSS-BBBC, CBO, 2D-CBO, SOS, SOS-ABF1, SOS-ABF2, SOS-ABF1\&2, and ISOS.

MSOS and SOS find Avg. solutions of 2185.4313 and $2303.3034 \mathrm{~kg}$, respectively. It is observed for the solutions that MSOS gives better Avg. solution than SOS and other solutions. MSOS and SOS find SD as15.4062 and 83.5897, respectively. It can be seen from the solutions that that MSOS finds better result as SD than SOS and other solutions presented in the table except SOS-ABF1. In addition, SOS-ABF1 and MSOS find SD nearly similar. However, few studies are available in the literature which demonstrate competitive edge to proposed algorithm like enhanced colliding bodies' optimization algorithm proposed by Kaveh and Ghazaan [83] gives Avg. and SD values of 2159.93 and $1.57 \mathrm{~kg}$, respectively, after 14,700 number of analyses, CSS-MBLS gives Avg. and SD values of 2,157.40 and $1.04 \mathrm{~kg}$, respectively, after 9600 number of analyses, AHEFA gives Avg. and SD values of 2161.0393 and $0.1783 \mathrm{~kg}$, respectively, after 11,300

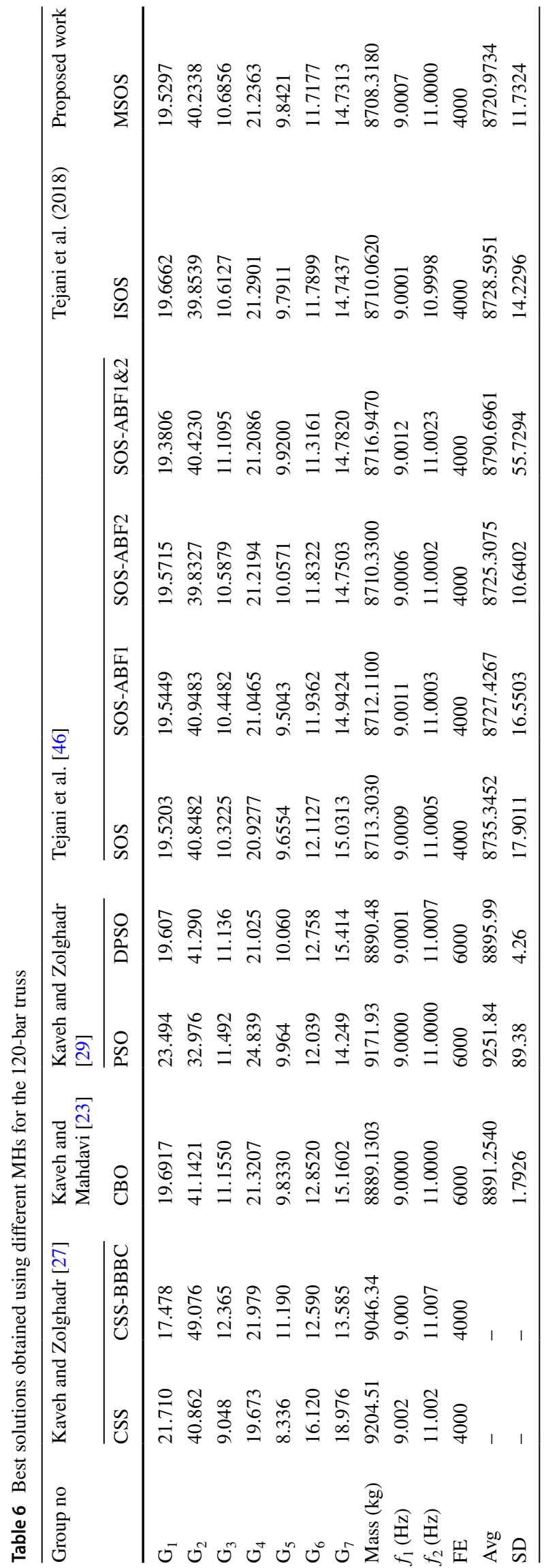




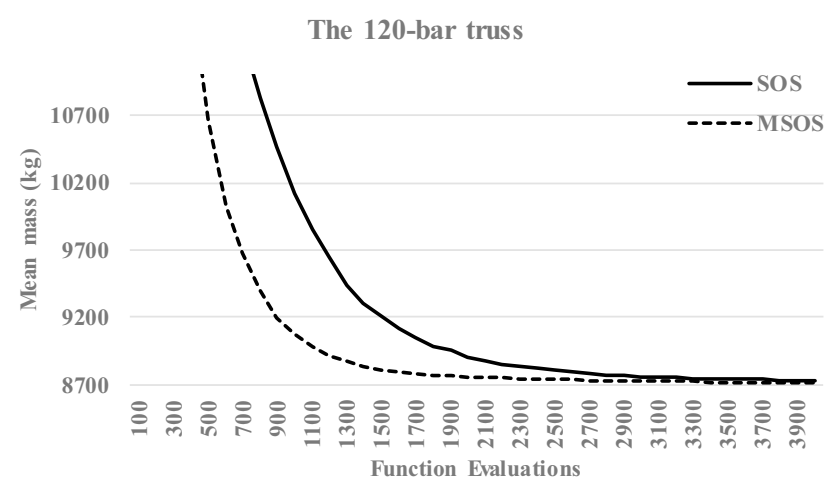

Fig. 12 Convergence curve of the 120-bar truss

function evaluation analyses, MC-TLBO gives Avg. and SD values of 2157.447 and $0.528 \mathrm{~kg}$, respectively, after 23,000 number of analyses.

This test clarifies that the solutions of MSOS are more reliable and proficient as compared to SOS and other solutions reported in literature. Figure 13 shows a convergence graph of the mean mass for MSOS and SOS of the 200-bar truss. It can be seen easily that the graph converges faster for initial 7000 FEs. The convergence graph and statistical results indicate that MSOS converges faster and set superior results.

The aforementioned discussion demonstrates the comparison between best mass, average mass, and SD values obtained using the proposed MSOS and SOS algorithms for six benchmark problems. In the tables, the average values exemplify the convergence rate of the algorithm, whereas the SD determines the search consistency. It can be seen from the result summary that performance of SOS has been improved by the proposed modifications. In addition, convergence plots represented above for benchmarks problems with proposed modification manifest the solution with faster convergence with minimum time. The overall performance of MSOS is the significant among the measured MHs. In addition, this study illustrates that the proposed modification outperformance with respect to the basic SOS algorithm. Obtained solutions confirm the merits of the proposed $\mathrm{MH}$.

\section{Corroborating MSOS performance with various benchmark functions}

This section the twenty-two benchmark functions are extracted from CEC 2014 Special Session and Competition on Real-Parameter Numerical Optimization (Liang et al. 2013) are exploited to manifest the effectiveness of the proposed algorithm. These twenty-two standard problems are epitomized in Table 8. Furthermore, these benchmark problems are subdivided into three segments: Three unimodal Functions (F1-F3), thirteen simple multimodal Functions (F4-F16) and also six hybrid Functions (F17-F22). Moreover, eight distinct optimization algorithms (viz. WWO, BA, HuS, GSA, BBO, IWO, SOS, and MSOS) has been applied to these standard problems and compared to verify the results. In this study, 30-dimensional functions are used with search ranges as $[-100,100]$. Population size is considered as 50 and $F E_{\max }$ are taken as 150,000 for proposed algorithm. All results are collected from 60 independent runs on each test function.

Comparative Avg. and SD of fitness values over the 60 runs are presented in Tables 9 and 10, respectively. Statistical tests are essential to check significance improvements by a proposed method over existing methods. Thus, the Friedman rank test on the results of MSOS, SOS, and other stateof-the-art algorithms. The test is performed on the Avg. and SD of functional values obtained. The tables also present the rank sum of the algorithms over the test functions. The results signify that MSOS outperforms other optimizers for unimodal functions followed by SOS and WWO. For multimodal and hybrid functions WWO gives best results followed by IWO and MSOS among the considered algorithms. Moreover, MSOS ranks better compared to SOS for unimodal, multimodal, and hybrid functions. The overall performance of MSOS is second best among the considered algorithms, whereas WWO performs the best on the benchmark functions of unimodal, multimodal, and hybrid functions. These results confirm the merits of the proposed algorithms once more.

\section{Conclusion and future perspectives}

A modified SOS is presented here for determining the minimum mass design of truss structure subjected to multiple natural frequency constraints with optimal nodal positions and element cross section areas. The proposed algorithm is applied successfully on five benchmark problems of simultaneous size and shape, optimization to investigate their performance. Complementarily, three unimodal functions, thirteen multimodal functions, and six hybrid functions of the CEC2014 are also investigated. A modified parasite vector is proposed here for improvement in exploitation capability of parasitism phase in basic SOS algorithm. Furthermore, an adaptive factor is introduced in basic SOS algorithm to improve its efficiency for complex structures. Motive behind proposed study is to maintain harmony between exploration and exploitation potency of optimization algorithm in search space. Here all benchmark problems are examined by considering constraints like natural 


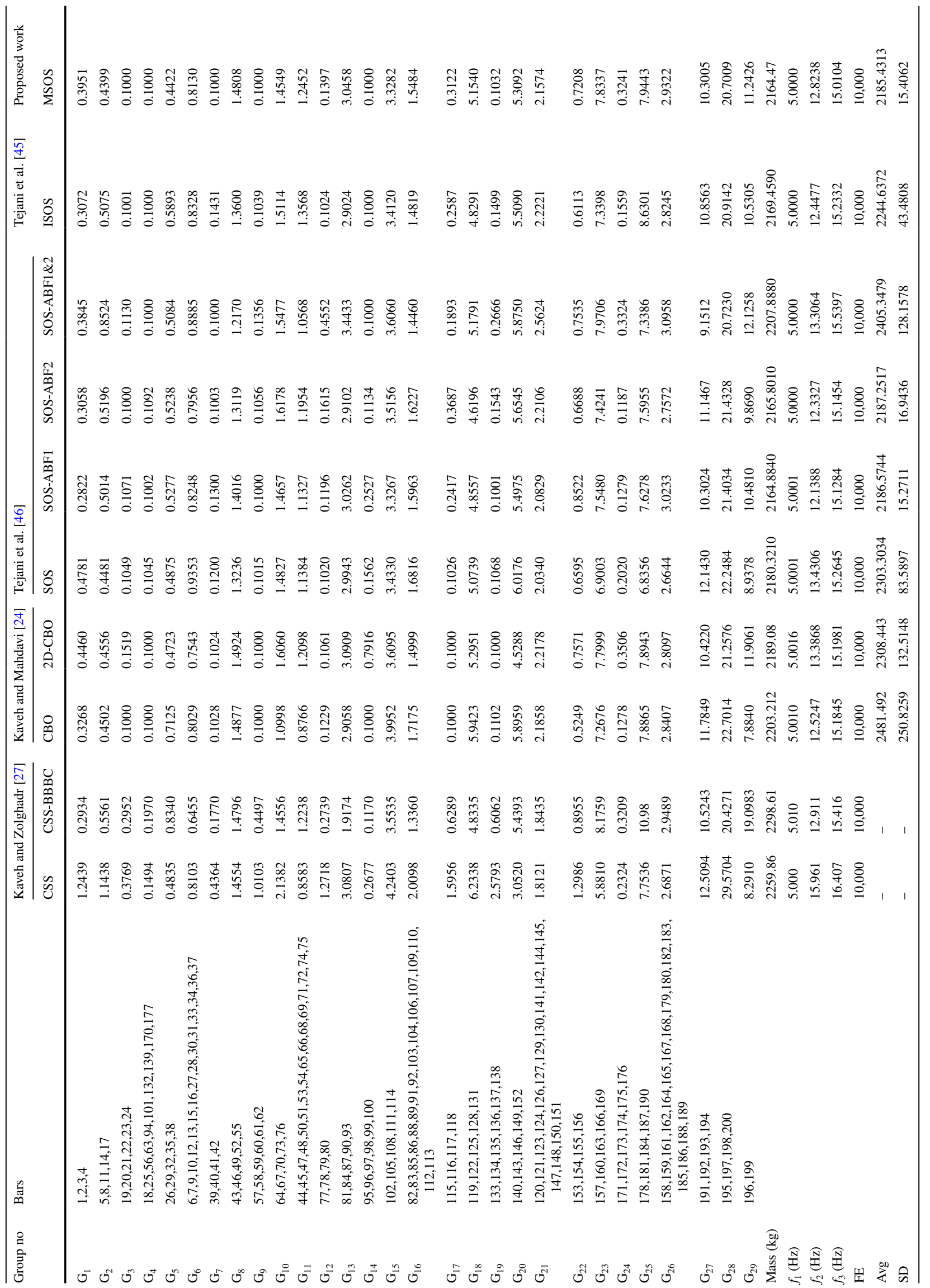




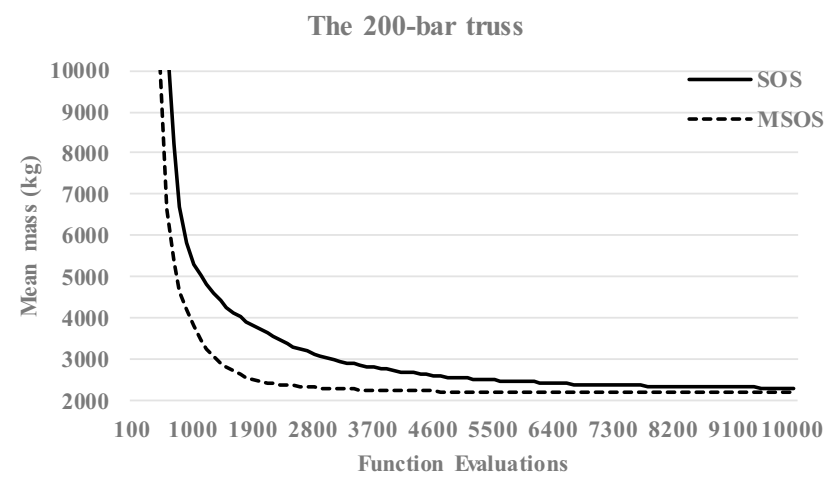

Fig. 13 Convergence curve of the 200-bar truss

frequency, nodal coordinates, and element section area for evaluation of feasibility and effectiveness of the algorithm. Usually design variable like nodal coordinates and element section area are of diverse nature and on collaboration they show complexity and diversified characteristics. In addition, frequency bounds with multiple design variable results into non-linearity and non-convexity of optimization problem.

In this study, the performance of the MSOS algorithm is compared with various existing MHs like NHGA, PSO, NHPGA, CSS, enhanced CSS, HS, FA, CSS-BBBC, hybrid OC-GA, TWO, SOS, SOS-ABF1, SOS-ABF2, SOS-ABF1\&2, and ISOS. Results shows the dominance of MSOS with respect to existing algorithms in parameters like optimum mass, Average mass, and SD of mass. Results also manifest that the combined utilization of adaptive factor and modification in parasitism vector in the proposed algorithm, improves the efficiency of search with good balance between exploration and exploitation potential. In addition, results show that the propose algorithm has high exploration potential during initial function evaluations and also high exploitation capability during remaining function evaluation, which shows its outstanding possession of global exploration and exploitation capacity in search space. To evaluate the performance of the proposed algorithms in benchmark functions, the results of SOS and MSOS are compared with the results of the WWO, BA, HuS, GSA, BBO, and IWO algorithms for the twenty-two benchmark functions proposed in the CEC2014 competition. Overall, MSOS has a better or competitive for obtaining results based on the average and $\mathrm{SD}$ of functional values obtained over the stated runs as compared to SOS.

A possible direction for future work would be to extend the proposed method to investigate the multi-objective truss structure design problems, where other objective functions such as joint cost and total construction cost can be taken into account. Application of efficient analysis techniques can facilitate the optimization problem of large-scale structures. It seems that introducing new mathematical and MHs as well as hybridizing and improving the existing ones to address structural optimization with frequency constraints will continue to grow as an active research topic. Moreover, utilization of novel algebraic and graph theoretical methods to decrease the computational time for optimization of different types of regular and near-regular structures can also be a very interesting field for future research. Considering frequency constraints along with stress, displacement, and other types of constraints can also receive more attraction. Inquisitive researchers can analyse this algorithm for various optimization problems, from small-scale engineering design problems to large-scale truss structure design

Table 8 CEC 201422 benchmark functions

\begin{tabular}{|c|c|c|c|}
\hline Test function & Optimum & Test function & Optimum \\
\hline F1: rotated high conditioned Elliptic function & 100 & F12: Shifted and Rotated Katsuura Function & 1200 \\
\hline F2: rotated bent Cigar function & 200 & F13: Shifted and Rotated HappyCat Function & 1300 \\
\hline F3: rotated discus function & 300 & F14: Shifted and Rotated HGBat Function & 1400 \\
\hline F4: shifted and rotated Rosenbrock's function & 400 & $\begin{array}{l}\text { F15: Shifted and Rotated Expanded Griewank's plus Rosen- } \\
\text { brock's Function }\end{array}$ & 1500 \\
\hline F5: Shifted and Rotated Ackley's Function & 500 & F16: Shifted and Rotated Expanded Scaffer's F6 Function & 1600 \\
\hline F6: Shifted and Rotated Weierstrass Function & 600 & F17: Hybrid Function 1 ((F9, F8,F1) & 1700 \\
\hline F7: Shifted and Rotated Griewank's Function & 700 & F18: Hybrid Function 2 (F2, F12, F8) & 1800 \\
\hline F8: Shifted Rastrigin's Function & 800 & F19: Hybrid Function 3 (F7, F6, F4, F14) & 1900 \\
\hline F9: Shifted and Rotated Rastrigin's Function & 900 & F20: Hybrid Function 4 (F12, F3, F13, F8) & 2000 \\
\hline F10: Shifted Schwefel's Function & 1000 & F21: Hybrid Function 5 (F14, F12, F4, F9, F1) & 2100 \\
\hline F11: Shifted and Rotated Schwefel's Function & 1100 & F22: Hybrid Function 6 (F10, F11, F13, F9, F5) & 2200 \\
\hline
\end{tabular}


Table 9 Comparative Avg. of fitness values of the CEC2014 (The results of first six algorithms are as per Zheng [76])

\begin{tabular}{|c|c|c|c|c|c|c|c|c|}
\hline Function & WWO & BA & Hus & GSA & $\mathrm{BBO}$ & IWO & SOS & MSOS \\
\hline $\mathrm{F} 1$ & 628064.7331 & 316593399.3261 & 5555804.7723 & 14413625.1299 & 27262607.1812 & 1463430.6695 & 1026753.8150 & 467711.3550 \\
\hline $\mathrm{F} 2$ & 330.4397 & 25714756385.1935 & 10068.1285 & 8771.2239 & 4012004.0764 & 17672.1722 & 213.1503 & 231.0785 \\
\hline F3 & 526.8209 & 72001.7161 & 502.0203 & 45384.2492 & 13100.3120 & 8167.4657 & 938.9390 & 496.9134 \\
\hline $\begin{array}{c}\text { Friedman } \\
\text { value of } \\
\text { F1-F3 }\end{array}$ & 8 & 24 & 12 & 17 & 20 & 15 & 8 & 4 \\
\hline $\begin{array}{c}\text { Friedman } \\
\text { rank of } \\
\text { F1-F3 }\end{array}$ & 2 & 8 & 4 & 6 & 7 & 5 & 2 & 1 \\
\hline F4 & 417.0105 & 3697.5439 & 506.9362 & 676.4360 & 538.7936 & 500.3255 & 468.2918 & 466.2221 \\
\hline F5 & 519.9999 & 520.9716 & 520.7029 & 519.9990 & 520.1556 & 520.0140 & 520.5639 & 520.0303 \\
\hline F6 & 605.9873 & 636.3693 & 623.0650 & 619.5872 & 613.9623 & 602.2138 & 610.8746 & 611.5147 \\
\hline F7 & 700.0037 & 910.6678 & 700.0407 & 700.0001 & 701.0283 & 700.0337 & 700.0161 & 700.0216 \\
\hline F8 & 801.1436 & 1070.3076 & 940.1063 & 800.4991 & 877.4573 & 843.7475 & 852.1217 & 824.6915 \\
\hline F9 & 961.0930 & 1250.0944 & 1011.9988 & 1059.7399 & 951.4286 & 946.0714 & 970.5093 & 966.3686 \\
\hline F10 & 1581.5778 & 6426.1095 & 2253.5001 & 4392.2443 & 1002.1744 & 2565.2591 & 2107.2343 & 1378.4436 \\
\hline F11 & 3349.4633 & 8152.1644 & 3302.9108 & 5099.2681 & 3247.3542 & 2887.3064 & 4017.4845 & 3636.8347 \\
\hline F12 & 1200.0995 & 1202.5771 & 1200.1870 & 1200.0011 & 1200.2257 & 1200.0355 & 1200.6611 & 1200.3483 \\
\hline F13 & 1300.2617 & 1304.0199 & 1300.3921 & 1300.2972 & 1300.5091 & 1300.2789 & 1300.4233 & 1300.3700 \\
\hline F14 & 1400.2169 & 1473.1361 & 1400.2377 & 1400.2540 & 1400.4439 & 1400.2360 & 1400.3309 & 1400.2643 \\
\hline F15 & 1503.2828 & 194533.2621 & 1517.0308 & 1503.2887 & 1514.6242 & 1503.6932 & 1517.6988 & 1510.9396 \\
\hline F16 & 1610.4351 & 1612.9981 & 1611.7074 & 1613.6691 & 1609.9125 & 1610.4324 & 1610.6564 & 1610.4357 \\
\hline $\begin{array}{c}\text { Friedman } \\
\text { value of } \\
\text { F4-F16 }\end{array}$ & 28 & 103 & 70 & 55 & 59 & 36 & 66 & 51 \\
\hline $\begin{array}{c}\text { Friedman } \\
\text { rank of } \\
\text { F4-F16 }\end{array}$ & 1 & 8 & 7 & 4 & 5 & 2 & 6 & 3 \\
\hline F17 & 26618.6801 & 4641277.7674 & 198099.0415 & 578588.7550 & 4299306.6650 & 86437.0037 & 143235.1725 & 81339.7737 \\
\hline F18 & 2026.3758 & 121880897.9466 & 3780.5580 & 2289.6856 & 28418.2340 & 5787.0752 & 8320.0810 & 5807.7762 \\
\hline F19 & 1907.7291 & 2004.9297 & 1931.0413 & 1995.2919 & 1928.4718 & 1907.9130 & 1923.3954 & 1920.0385 \\
\hline F20 & 5363.8611 & 19356.8922 & 38657.3368 & 22421.9064 & 31411.1843 & 2992.6053 & 5770.2949 & 5697.8995 \\
\hline F21 & 38673.7809 & 1095231.5294 & 60455.7923 & 170612.9594 & 485593.2936 & 39074.3102 & 68597.8240 & 32529.2852 \\
\hline F22 & 2481.9864 & 3134.0717 & 3072.5807 & 3161.1458 & 2722.8879 & 2346.3986 & 2496.3689 & 2483.6063 \\
\hline $\begin{array}{l}\text { Friedman } \\
\text { value } \\
\text { of F17- } \\
\text { F22 }\end{array}$ & 9 & 44 & 32 & 35 & 38 & 14 & 27 & 17 \\
\hline $\begin{array}{l}\text { Friedman } \\
\text { rank of } \\
\text { F17- } \\
\text { F22 }\end{array}$ & 1 & 8 & 5 & 6 & 7 & 2 & 4 & 3 \\
\hline $\begin{array}{l}\text { Overall } \\
\text { Fried- } \\
\text { man } \\
\text { value }\end{array}$ & 45 & 171 & 114 & 107 & 117 & 65 & 101 & 72 \\
\hline $\begin{array}{l}\text { Overall } \\
\text { Fried- } \\
\text { man } \\
\text { rank }\end{array}$ & 1 & 8 & 6 & 5 & 7 & 2 & 4 & 3 \\
\hline
\end{tabular}


Table 10 Comparative SD of fitness values of the CEC2014 The results of first six algorithms are as per Zheng [76]

\begin{tabular}{|c|c|c|c|c|c|c|c|c|}
\hline Function & WWO & BA & Hus & GSA & $\mathrm{BBO}$ & IWO & SOS & MSOS \\
\hline F1 & 244526.8140 & 104690309.2627 & 2620084.7953 & 13187933.1609 & 16720012.8760 & 571747.0082 & 732930.2258 & 255076.7193 \\
\hline $\mathrm{F} 2$ & 202.2221 & 7553596375.4800 & 6012.6897 & 2903.3044 & 1549219.3214 & 8673.4818 & 20.2822 & 63.3820 \\
\hline F3 & 184.6450 & 17548.6717 & 540.6109 & 10432.6453 & 12764.8742 & 2692.8884 & 527.5818 & 249.8799 \\
\hline $\mathrm{F} 4$ & 36.3636 & 1973.8532 & 36.6181 & 51.5149 & 38.3545 & 28.7968 & 31.7519 & 40.9633 \\
\hline F5 & 0.0007 & 0.0481 & 0.0783 & 0.0006 & 0.0422 & 0.0038 & 0.0801 & 0.0430 \\
\hline F6 & 2.6204 & 1.5591 & 2.1784 & 1.8319 & 2.3542 & 1.1219 & 2.5681 & 3.0635 \\
\hline F7 & 0.0063 & 32.3193 & 0.0556 & 0.0010 & 0.0264 & 0.0121 & 0.0214 & 0.0271 \\
\hline F8 & 2.3361 & 25.6476 & 12.7304 & 0.2063 & 20.6917 & 10.1117 & 12.3208 & 6.9815 \\
\hline F9 & 11.0977 & 44.1294 & 25.9919 & 17.4329 & 11.4372 & 11.3933 & 24.0796 & 16.9302 \\
\hline F10 & 361.6122 & 518.6548 & 433.1531 & 360.9861 & 0.6800 & 380.0190 & 344.1052 & 187.9776 \\
\hline F11 & 289.2180 & 362.2389 & 465.5429 & 567.3467 & 511.5523 & 447.7160 & 835.0838 & 506.0594 \\
\hline F12 & 0.0561 & 0.3339 & 0.0777 & 0.0010 & 0.0562 & 0.0148 & 0.1833 & 0.1351 \\
\hline F13 & 0.0641 & 0.5483 & 0.0650 & 0.0665 & 0.1061 & 0.0650 & 0.0864 & 0.0783 \\
\hline F14 & 0.0441 & 13.9463 & 0.0474 & 0.0423 & 0.1992 & 0.1191 & 0.1296 & 0.0538 \\
\hline F15 & 0.7753 & 140338.9490 & 3.2695 & 0.7297 & 4.2976 & 0.8484 & 3.7981 & 4.1851 \\
\hline F16 & 0.4667 & 0.1904 & 0.7249 & 0.3428 & 0.5923 & 0.6144 & 0.6059 & 0.7675 \\
\hline F17 & 12403.5374 & 1789909.2516 & 160518.8631 & 219949.3460 & 4192494.2708 & 68473.6644 & 159023.3392 & 54940.7907 \\
\hline F18 & 125.1962 & 100285357.3457 & 2246.5148 & 377.9286 & 19674.9440 & 3690.0554 & 10313.3555 & 5702.6751 \\
\hline F19 & 1.3780 & 20.3164 & 33.1485 & 34.3190 & 27.6885 & 1.6545 & 26.6142 & 22.3884 \\
\hline F20 & 3177.0847 & 10283.6255 & 8492.7252 & 13860.3564 & 17604.9005 & 700.4102 & 3295.2452 & 2466.1799 \\
\hline F21 & 35555.5716 & 750680.8765 & 42428.1036 & 65285.4119 & 334571.5390 & 23011.1766 & 80093.3096 & 24345.4802 \\
\hline F22 & 142.8952 & 205.4095 & 267.2685 & 250.0137 & 234.4393 & 73.3907 & 151.5147 & 141.3538 \\
\hline $\begin{array}{l}\text { Overall } \\
\text { Fried- } \\
\text { man } \\
\text { value }\end{array}$ & 51.0000 & 144 & 113.5 & 89 & 128 & 67.5 & 108 & 91 \\
\hline $\begin{array}{l}\text { Overall } \\
\text { Fried- } \\
\text { man } \\
\text { rank }\end{array}$ & 1 & 8 & 6 & 3 & 7 & 2 & 5 & 4 \\
\hline
\end{tabular}

problems. Moreover, one can attempt to resolve the persistence problem of tuning of unpredictable parameters in algorithms.

\section{References}

1. Baykasoğlu A, Ozsoydan FB (2015) Adaptive firefly algorithm with chaos for mechanical design optimization problems. Appl Soft Comput 36:152-164. https://doi.org/10.1016/j. asoc.2015.06.056

2. Bingul $Z$ (2007) Adaptive genetic algorithms applied to dynamic multiobjective problems. Appl Soft Comput J 7:791-799. https:// doi.org/10.1016/j.asoc.2006.03.001

3. Cheng MY, Prayogo D (2014) Symbiotic Organisms Search: A new metaheuristic optimization algorithm. Comput Struct 139:98-112. https://doi.org/10.1016/j.compstruc.2014.03.007

4. Cheng MY, Prayogo D, Wu YW (2018) Prediction of permanent deformation in asphalt pavements using a novel symbiotic organisms search - least squares support vector regression. Neural Comput Appl. https://doi.org/10.1007/s00521-018-3426-0
5. De Jong KA (1975) An Analysis of the Behavior of a Class of Genetic Adaptive Systems. Comput Commun Sci 56:266

6. Do DTT, Lee J (2017) A modified symbiotic organisms search (mSOS) algorithm for optimization of pin-jointed structures. Appl Soft Comput J 61:683-699. https://doi.org/10.1016/j. asoc.2017.08.002

7. Dorigo M, Maniezzo V, Colorni A (1996) Ant System: Optimization by a Colony of Cooperating Agents. IEEE Trans Syst Man Cybern Part B 26:1-13. https://doi.org/10.1109/3477.484436

8. Eberhart R, Kennedy J (1995) A new optimizer using particle swarm theory. MHS'95 Proc Sixth Int Symp Micro Mach Hum Sci 39-43. https://doi.org/10.1109/MHS.1995.494215

9. Erol OK, Eksin I (2006) A new optimization method: Big Bang-Big Crunch. Adv Eng Softw 37:106-111. https://doi. org/10.1016/j.advengsoft.2005.04.005

10. Eskandar H, Sadollah A, Bahreininejad A, Hamdi M (2012) Water cycle algorithm - a novel metaheuristic optimization method for solving constrained engineering optimization problems. Comput Struct 110-111:151-166. https://doi.org/10.1016/j.comps truc.2012.07.010

11. Ezugwu AES, Adewumi AO, Frîncu ME (2017) Simulated annealing based symbiotic organisms search optimization algorithm for 
traveling salesman problem. Expert Syst Appl 77:189-210. https ://doi.org/10.1016/j.eswa.2017.01.053

12. Farshchin M, Camp CV, Maniat M (2016) Multi-class teachinglearning-based optimization for truss design with frequency constraints. Eng Struct 106:355-369. https://doi.org/10.1016/j.engst ruct.2015.10.039

13. Fogel DB (1988) An Evolutionary Approach to the Traveling Salesman Problem. Biol Cybern 60:139-144. https://doi. org/10.1007/BF00202901

14. Glover F (1975) Tabu search and adaptive memory programming —advances, applications and challenges. In: In Interfaces in computer science and operations research. pp 1-75

15. Goldberg DE, Holland JH (1988) Genetic algorithms and machine learning. Mach Learn 3:95-99. https://doi. org/10.1023/A:1022602019183

16. Gomes HM (2011) Truss optimization with dynamic constraints using a particle swarm algorithm. Expert Syst Appl 38:957968. https://doi.org/10.1016/j.eswa.2010.07.086

17. Karaboga D, Basturk B (2007) A powerful and efficient algorithm for numerical function optimization: Artificial bee colony (ABC) algorithm. J Glob Optim 39:459-471. https://doi. org/10.1007/s10898-007-9149-x

18. Kaveh A, Farhoudi N (2013) A new optimization method: Dolphin echolocation. Adv Eng Softw 59:53-70. https://doi. org/10.1016/j.advengsoft.2013.03.004

19. Kaveh A, Ilchi Ghazaan M (2015) Hybridized optimization algorithms for design of trusses with multiple natural frequency constraints. Adv Eng Softw 79:137-147. https://doi. org/10.1016/j.advengsoft.2014.10.001

20. Kaveh A, Ilchi Ghazaan M (2017) Vibrating particles system algorithm for truss optimization with multiple natural frequency constraints. Acta Mech 228:307-322. https://doi.org/10.1007/ s00707-016-1725-z

21. Kaveh A, Jafari L, Farhoudi N (2015) Truss optimization with natural frequency constraints using a dolphin echolocation algorithm. Asian J Civ Eng 16:29-46

22. Kaveh A, Khayatazad M (2013) Ray optimization for size and shape optimization of truss structures. Comput Struct 117:8294. https://doi.org/10.1016/j.compstruc.2012.12.010

23. Kaveh A, Mahdavi VR (2014) Colliding bodies optimization: A novel meta-heuristic method. Comput Struct 139:18-27. https ://doi.org/10.1016/j.compstruc.2014.04.005

24. Kaveh A, Mahdavi VR (2015) Two-dimensional colliding bodies algorithm for optimal design of truss structures. Adv Eng Softw 83:70-79. https://doi.org/10.1016/j.advengsoft.2015.01.007

25. Kaveh A, Talatahari S (2010) A novel heuristic optimization method: Charged system search. Acta Mech 213:267-289. https ://doi.org/10.1007/s00707-009-0270-4

26. Kaveh A, Zolghadr A (2011) Shape and size optimization of truss structures with frequency constraints using enhanced charged system search algorithm. Asian J Civ Eng 12:487-509

27. Kaveh A, Zolghadr A (2012) Truss optimization with natural frequency constraints using a hybridized CSS-BBBC algorithm with trap recognition capability. Comput Struct 102-103:14-27. https ://doi.org/10.1016/j.compstruc.2012.03.016

28. Kaveh A, Zolghadr A (2013) Topology optimization of trusses considering static and dynamic constraints using the CSS. Appl Soft Comput J 13:2727-2734. https://doi.org/10.1016/j. asoc.2012.11.014

29. Kaveh A, Zolghadr A (2014) Democratic PSO for truss layout and size optimization with frequency constraints. Comput Struct 130:10-21. https://doi.org/10.1016/j.compstruc.2013.09.002

30. Kaveh A, Zolghadr A (2017) Truss shape and size optimization with frequency constraints using Tug of War Optimization. Asian J Civ Eng 18:311-313
31. Kirkpatrick S, Gelatt CD, Vecchi MP (1983) Optimization by Simulated Annealing. Science 220:671-680. https://doi.org/10.1126/ science.220.4598.671

32. Koza JR (1994) Genetic programming as a means for programming computers by natural selection. Stat Comput 4:87-112. https ://doi.org/10.1007/BF00175355

33. Lee KS, Geem ZW (2004) A new structural optimization method based on the harmony search algorithm. Comput Struct 82:781798. https://doi.org/10.1016/j.compstruc.2004.01.002

34. Li X, Yin M (2015) Modified cuckoo search algorithm with self adaptive parameter method. Inf Sci (Ny) 298:80-97. https://doi. org/10.1016/j.ins.2014.11.042

35. Liao TW, Kuo RJ (2018) Five discrete symbiotic organisms search algorithms for simultaneous optimization of feature subset and neighborhood size of KNN classification models. Appl Soft Comput J 64:581-595. https://doi.org/10.1016/j.asoc.2017.12.039

36. Miguel LFF, Fadel Miguel LF (2012) Shape and size optimization of truss structures considering dynamic constraints through modern metaheuristic algorithms. Expert Syst Appl 39:9458-9467. https://doi.org/10.1016/j.eswa.2012.02.113

37. Mortazavi A, Toğan V (2017) Sizing and layout design of truss structures under dynamic and static constraints with an integrated particle swarm optimization algorithm. Appl Soft Comput J 51:239-252. https://doi.org/10.1016/j.asoc.2016.11.032

38. Noilublao N, Bureerat S (2011) Simultaneous topology, shape and sizing optimisation of a three-dimensional slender truss tower using multiobjective evolutionary algorithms. Comput Struct 89:2531-2538. https://doi.org/10.1016/j.compstruc.2011.08.010

39. Osman IH, Laporte G (1996) Metaheuristics: A bibliography. Ann Oper Res 63:511-623. https://doi.org/10.1007/BF02125421

40. Patel V, Savsani V (2014) Optimization of a plate-fin heat exchanger design through an improved multi-objective teachinglearning based optimization (MO-ITLBO) algorithm. Chem Eng Res Des 92:2371-2382. https://doi.org/10.1016/j.cherd .2014.02.005

41. Piotrowski AP (2013) Adaptive memetic differential evolution with global and local neighborhood-based mutation operators. Inf Sci (Ny) 241:164-194. https://doi.org/10.1016/j.ins.2013.03.060

42. Savsani VJ, Tejani GG, Patel VK (2016) Truss topology optimization with static and dynamic constraints using modified subpopulation teaching-learning-based optimization. Eng Optim 48:1990-2006. https://doi.org/10.1080/0305215X.2016.1150468

43. Savsani VJ, Tejani GG, Patel VK, Savsani P (2017) Modified meta-heuristics using random mutation for truss topology optimization with static and dynamic constraints. J Comput Des Eng 4:106-130. https://doi.org/10.1016/j.jcde.2016.10.002

44. Shan H, Yasuda T, Ohkura K (2015) A self adaptive hybrid enhanced artificial bee colony algorithm for continuous optimization problems. BioSystems 132-133:43-53. https://doi. org/10.1016/j.biosystems.2015.05.002

45. Tejani GG, Savsani VJ, Bureerat S, Patel VK (2018) Topology and Size Optimization of Trusses with Static and Dynamic Bounds by Modified Symbiotic Organisms Search. J Comput Civ Eng 32:1-11. https://doi.org/10.1061/(ASCE)CP.1943-5487.0000741

46. Tejani GG, Savsani VJ, Patel VK (2016) Adaptive symbiotic organisms search (SOS) algorithm for structural design optimization. J Comput Des Eng 3:226-249. https://doi.org/10.1016/j. jcde.2016.02.003

47. Tejani GG, Savsani VJ, Patel VK (2016) Modified sub-population teaching-learning-based optimization for design of truss structures with natural frequency constraints. Mech Based Des Struct Mach 44:495-513. https://doi.org/10.1080/15397734.2015.1124023

48. Tejani GG, Savsani VJ, Patel VK, Bureerat S (2017) Topology, shape, and size optimization of truss structures using modified teaching-learning based optimization. Adv Comput Des 2:313331. https://doi.org/10.12989/acd.2017.2.4.313 
49. Tollo G di, Lardeux F, Maturana J, Saubion F (2015) An experimental study of adaptive control for evolutionary algorithms. Appl Soft Comput 35:359-372. https://doi.org/10.1016/j. asoc.2015.06.016

50. Tran DH, Cheng MY, Prayogo D (2016) A novel Multiple Objective Symbiotic Organisms Search (MOSOS) for time-cost-labor utilization tradeoff problem. Knowledge-Based Syst 94:132-145. https://doi.org/10.1016/j.knosys.2015.11.016

51. Tran DH, Luong-Duc L, Duong MT et al (2018) Opposition multiple objective symbiotic organisms search (OMOSOS) for time, cost, quality and work continuity tradeoff in repetitive projects. J Comput Des Eng 5:160-172. https://doi.org/10.1016/j. jcde.2017.11.008

52. Tejani GG, Pholdee N, Bureerat S, Prayogo D (2018) Multiobjective adaptive symbiotic organisms search for truss optimization problems. Knowl-Based Syst. https://doi.org/10.1016/j.knosy s.2018.08.005

53. Wang D, Zhang WH, Jiang JS (2004) Truss optimization on shape and sizing with frequency constraints. AIAA J 42:622-630. https ://doi.org/10.2514/1.1711

54. Wei L, Tang T, Xie X, Shen W (2011) Truss optimization on shape and sizing with frequency constraints based on parallel genetic algorithm. Struct Multidiscip Optim 43:665-682

55. Wolpert DH, Macready WG (1997) No free lunch theorems for optimization. IEEE Trans Evol Comput 1:67-82. https://doi. org/10.1109/4235.585893

56. Yang XS (2009) Firefly Algorithms for Multimodal Optimization. In: In International symposium on stochastic algorithms. pp 169-178

57. Yang XS, Deb S (2009) Cuckoo search via Levy flights. In: 2009 World Congress on Nature and Biologically Inspired Computing, NABIC 2009 - Proceedings. pp 210-214

58. Yu VF, Redi AANP, Yang CL et al (2017) Symbiotic organisms search and two solution representations for solving the capacitated vehicle routing problem. Appl Soft Comput J 52:657-672. https://doi.org/10.1016/j.asoc.2016.10.006

59. Zuo W, Bai J, Li B (2014) A hybrid OC-GA approach for fast and global truss optimization with frequency constraints. Appl Soft Comput J 14:528-535. https://doi.org/10.1016/j.asoc.2013.09.002

60. Cheng M-Y, Prayogo D, Tran D-H (2016) Optimizing MultipleResources Leveling in Multiple Projects Using Discrete Symbiotic Organisms Search. J Comput Civ Eng 30:04015036. https://doi. org/10.1061/(ASCE)CP.1943-5487.0000512

61. Duman S (2017) Symbiotic organisms search algorithm for optimal power flow problem based on valve-point effect and prohibited zones. Neural Comput Appl 28:3571-3585. https://doi. org/10.1007/s00521-016-2265-0

62. Prasad D, Mukherjee V (2016) A novel symbiotic organisms search algorithm for optimal power flow of power system with FACTS devices. Eng Sci Technol Int J 19:79-89. https://doi. org/10.1016/j.jestch.2015.06.005

63. Abdullahi M, Ngadi MA, Abdulhamid SM (2016) Symbiotic Organism Search optimization based task scheduling in cloud computing environment. Futur Gener Comput Syst 56:640-650. https://doi.org/10.1016/j.future.2015.08.006

64. Panda A, Pani S (2016) A Symbiotic Organisms Search algorithm with adaptive penalty function to solve multi-objective constrained optimization problems. Appl Soft Comput J 46:344-360. https://doi.org/10.1016/j.asoc.2016.04.030

65. Panda A, Pani S (2017) An orthogonal parallel symbiotic organism search algorithm embodied with augmented Lagrange multiplier for solving constrained optimization problems. Soft Comput doi. https://doi.org/10.1007/s00500-017-2693-5

66. Prayogo D (2018) Optimization model for construction project resource leveling using a novel modified symbiotic organisms search. Asian J Civ Eng 3456789:. https://doi.org/10.1007/s4210 7-018-0048-x

67. Subhabrata Banerjee SC (2017) Power Optimization of Three Dimensional Turbo Code Using a Novel Modified Symbiotic Organism Search. Wirel Pers Commun doi. https://doi. org/10.1007/s11277-016-3586-0

68. Guha D, Kumar P, Banerjee S (2018) Symbiotic organism search algorithm applied to load frequency control of multi-area power system. Energy Syst. https://doi.org/10.1007/s12667-017-0232-1

69. Dosoglu MK, Guvenc U, Duman S, Sonmez Y (2018) Symbiotic organisms search optimization algorithm for economic / emission dispatch problem in power systems. Neural Comput Appl. https://doi.org/10.1007/s00521-016-2481-7

70. Saha S, Mukherjee V (2016) Optimal placement and sizing of DGs in RDS using chaos embedded SOS algorithm. 3671-3680. https://doi.org/10.1049/iet-gtd.2016.0151

71. Zhou Y, Wu H, Luo Q, Abdel-baset M (2018) Automatic data clustering using nature-inspired symbiotic organism search algorithm. Knowledge-Based Syst. https://doi.org/10.1016/j. knosys.2018.09.013

72. Prayogo D, Cheng MY, Prayogo H (2017) A Novel implementation of nature-inspired optimization for civil engineering: a comparative study of symbiotic organisms search. 19:36-43. https:// doi.org/10.9744/CED.19.1.36-43

73. Jaffel Z, Farah M (2018, March) A symbiotic organisms search algorithm for feature selection in satellite image classification. In: Advanced Technologies for Signal and Image Processing (ATSIP), 2018 4th International Conference on(pp. 1-5). IEEE

74. Sulaiman M, Ahmad A, Khan A, Muhammad S (2018) Hybridized symbiotic organism search algorithm for the optimal operation of directional overcurrent relays. Hindawi Complex 2018:11. https ://doi.org/10.1155/2018/4605769

75. Tejani GG, Savsani VJ, Patel VK, Mirjalili S (2018) KnowledgeBased Systems Truss optimization with natural frequency bounds using improved symbiotic organisms search. Knowledge-Based Syst 5:1-17. https://doi.org/10.1016/j.knosys.2017.12.012

76. Zheng Y (2015) Computers \& Operations Research Water wave optimization: A new nature-inspired metaheuristic. Comput Oper Res 55:1-11. https://doi.org/10.1016/j.cor.2014.10.008

77. Črepinšek M, Liu S-H, Mernik M (2013) Exploration and Exploitation in Evolutionary Algorithms: A Survey. ACM Comput 45:133. https://doi.org/10.1145/2480741.2480752

78. Geem Z, Kim J, Loganathan GV (2001) A New Heuristic Optimization Algorithm: Harmony Search. Simulation 76:60-68. https ://doi.org/10.1177/003754970107600201

79. Al-sharhan S (2016) An enhanced symbiosis organisms search algorithm: an empirical study. Neural Comput Appl doi. https:// doi.org/10.1007/s00521-016-2624-X

80. Liang JJ, Qu BY, Suganthan PN (2014) Problem Definitions and Evaluation Criteria for the CEC 2014 Special Session and Competition on Single Objective Real-Parameter Numerical Optimization

81. Grandhi R (1993) Structural Optimization with Frequency Constraints - A Review. AIAA J 31:2296-2303. https://doi. org $/ 10.2514 / 3.11928$

82. Kaveh A, Ilchi M, Taha G (2013) An improved ray optimization algorithm for design of truss structures. Period Polytech 2:97-112. https://doi.org/10.3311/PPci.7166

83. Kaveh A, Ghazaan MI (2014) Enhanced colliding bodies algorithm for truss optimization with frequency constraints. J Comput Civ Eng 29:1-11. https://doi.org/10.1061/(ASCE)CP.19435487.0000445

84. Miao F, Zhou Y, Luo Q (2018) A modified symbiotic organisms search algorithm for unmanned combat aerial vehicle route planning problem. J Oper Res Soc 5682:1-32. https://doi. org/10.1080/01605682.2017.1418151 
85. Bureerat S, Ph D, Pholdee N, Ph D (2015) Optimal Truss Sizing Using an Adaptive Differential Evolution Algorithm. J Comput Civ Eng. https://doi.org/10.1061/(ASCE)CP.1943-5487.0000487

86. Ou-yang C, Hanyata TB, Samadhi TMAA (2015) Hybrid selfadaptive-velocity particle swarm optimisation-Cooper heuristic for the facility location allocation problem in Jakarta. https://doi. org/10.1080/23302674.2015.1029565

87. Pham HA (2016) Advances in Engineering Software Truss optimization with frequency constraints using enhanced differential evolution based on adaptive directional mutation and nearest neighbor comparison. Adv Eng Softw 102:142-154. https://doi. org/10.1016/j.advengsoft.2016.10.004

88. Lieu QX, Do DTT, Lee J (2018) An adaptive hybrid evolutionary firefly algorithm for shape and size optimization of truss structures with frequency constraints. Comput Struct 195:99-112. https:// doi.org/10.1016/j.compstruc.2017.06.016

89. Ho-Huu V, Nguyen-Thoi T, Truong-Khac T, Le-Anh L, Vo-Duy T (2016) An improved differential evolution based on roulette wheel selection for shape and size optimization of truss structures with frequency constraints. Neural Comput Appl 29:167-185. https:// doi.org/10.1007/s00521-016-2426-1
90. Kaveh A, Javadi SM (2013) Shape and size optimization of trusses with multiple frequency constraints using harmony search and ray optimizer for enhancing the particle swarm optimization algorithm. 1605:1595-1605. https://doi.org/10.1007/s0070 7-013-1006-z

91. Kaveh A, Zolghadr A (2017) Cyclical parthenogenesis algorithm for layout optimization of truss structures with frequency constraints. Eng Optim 0:1-18. https://doi.org/10.1080/03052 15X.2016.1245730

92. Kaveh A, Zolghadr A (2014) A new PSRO algorithm for frequency constraint truss shape and size optimization. Struct Eng Mech 52:445-468. https://doi.org/10.12989/sem.2014.52.3.445

93. Jalili S, Talatahari S (2017) Optimum Design of Truss Structures Under Frequency Constraints using Hybrid CSS-MBLS Algorithm. 00:1-14. https://doi.org/10.1007/s12205-017-1407-y

Publisher's Note Springer Nature remains neutral with regard to jurisdictional claims in published maps and institutional affiliations. 